\title{
Segmentation of the Luminal Border in Intravascular Ultrasound B-mode Images Using a Probabilistic Approach
}

\author{
E. Gerardo Mendizabal-Ruiz ${ }^{\mathrm{a}}$, Mariano Rivera ${ }^{\mathrm{b}}$, Ioannis A. Kakadiaris ${ }^{\mathrm{a}, *}$ \\ ${ }^{a}$ Computational Biomedicine Lab, Departments of Computer Science, \\ Electrical \& Computer Engineering, and Biomedical Engineering, \\ University of Houston, Houston, TX, USA \\ ${ }^{b}$ Centro de Investigación en Matemáticas. Guanajuato, Gto. México
}

\begin{abstract}
Intravascular ultrasound (IVUS) is a catheter-based medical imaging technique that produces cross-sectional images of blood vessels and is particularly useful for studying atherosclerosis. In this paper, we present a computational method for the delineation of the luminal border in IVUS B-mode images. The method is based in the minimization of a probabilistic cost function (that deforms a parametric curve) which defines a probability field that is regularized with respect to the given likelihoods of the pixels belonging to blood and non-blood. These likelihoods are obtained by a Support Vector Machine classifier trained using samples of the lumen and non-lumen regions provided by the user in the first frame of the sequence to be segmented. In addition, an optimization strategy is introduced in which the direction of the steepest descent and Broyden-Fletcher-Goldfarb-Shanno optimization methods are linearly combined to improve convergence. Our proposed method (MRK) is capable of segmenting IVUS B-mode images from different systems and transducer frequencies without the need of any parameter tuning, and it is robust with respect to changes of the B-mode reconstruction parameters which are subjectively adjusted by the interventionist. We validated the proposed method on six $20 \mathrm{MHz}$ and six $40 \mathrm{MHz}$ IVUS stationary sequences corresponding to regions with different degrees of stenosis, and evaluated its performance by comparing the
\end{abstract}

\footnotetext{
*Corresponding author

Email address: ioannisk@uh. edu (Ioannis A. Kakadiaris )

${ }^{1} 501$ Philip Guthrie Hoffman Hall, 4800 Calhoun Houston, TX 77204, Phone: +1 (713) 7431255, Fax: +1 (713) 743-1250
} 
segmentation results with manual segmentation by two observers. Furthermore, we compared our method with the segmentation results on the same sequences as provided by the authors of three other segmentation methods available in the literature. The performance of all methods was quantified using Dice and Jaccard similarity indexes, Hausdorff distance, linear regression and Bland-Altman analysis. The results indicate the advantages of our method for the segmentation of the lumen contour.

Keywords: IVUS, Probabilistic Segmentation, Contour Parameterization, Ultrasound, Coronary Arteries

\section{Introduction}

Complications attributed to cardiovascular disease (CVD) are currently the main cause of death worldwide (Lloyd-Jones et al. (2009)). It is known that the majority of adverse CVD-related events are due to coronary artery disease, a condition in which fatty lesions called plaques are formed on the walls of those vessels which nourish the heart with blood. Sudden rupture of a plaque may lead to a rapidly-progressing stenotic condition in which the blood supply is entirely cut off from a region of the heart. This may result in death.

Intravascular ultrasound (IVUS) is a catheter-based medical imaging technique capable of providing high-resolution, cross-sectional images of the interior of blood vessels in real time allowing the collection of morphological information about the vessel. The IVUS catheter consists of either a solid-state or a mechanically-rotated transducer which transmits ultrasound pulses and receives a reflected signal (i.e., A-line) over each radial scan at a discrete set of angles. Commonly, 240 to 360 A-line signals are obtained and digitized per rotation. The envelopes of these signals are computed, compressed, stacked along the angular direction, and mapped into 8-bit gray scale to form an image known as a polar B-mode image. Finally, the polar B-mode image is geometrically transformed to obtain the familiar disc-shaped image known as a Cartesian B-mode image.

The IVUS imaging technique consists of steering a guidewire with a small diameter (usually $0.36 \mathrm{~mm}$ ) into the blood vessel branch to be imaged. The ultrasound catheter is then slid-in over the guidewire and positioned within a target segment of interest. The IVUS system starts acquiring and displaying the images usually at 30 frames/second. The ultrasound catheter is then pulled back in order to identify and analyze the vessel regions. The pullback can be performed either manually or using a motorized device which moves the transducer at a constant 


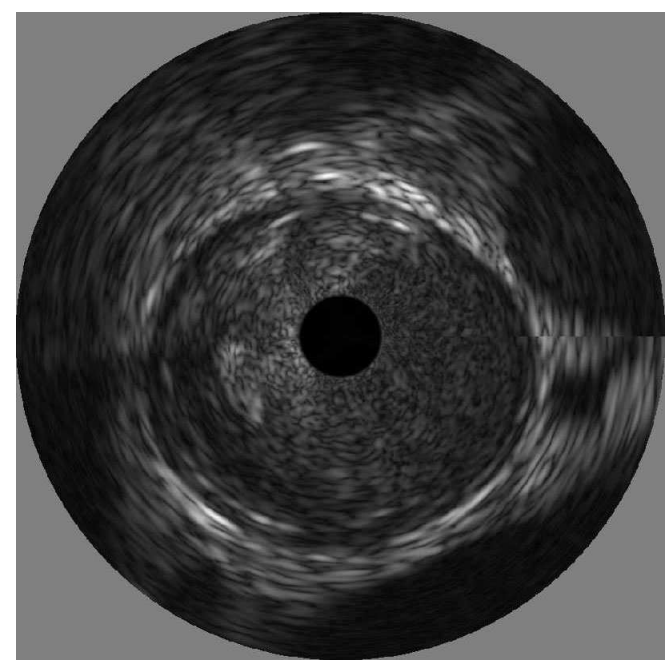

(a)

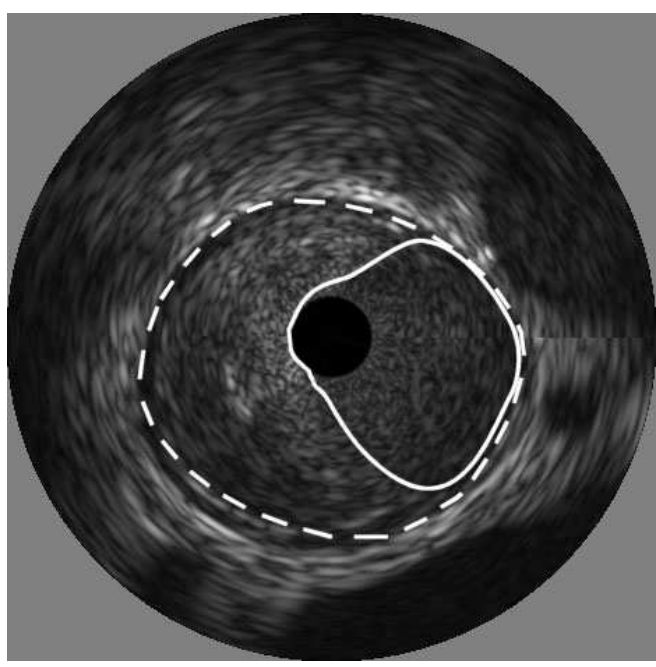

(b)

Figure 1: Example of (a) an typical IVUS image with (b) its corresponding segmentation (lumen/intima and media/adventitia interface are depicted using a solid and dotted lines, respectively).

determined speed (usually $0.5 \mathrm{~mm} / \mathrm{s}$ ). In human coronary arteries, the target segments generally include at least $10 \mathrm{~mm}$ of distal vessel, the lesion site(s), and the entire proximal vessel back to the aorta (Mintz et al. (2001)). If additional information about a lesion is needed, an acquisition may be performed by maintaining the IVUS catheter stationary over the region of interest. The analysis of the acquired data may be performed in real-time during the intervention or off-line after the acquisition.

Segmentation of IVUS images refers to the delineation of the lumen/intima and media/adventita borders (Fig. 1). This process is necessary for assessing morphological characteristics of the vessel and plaque such as lumen diameter, minimum lumen cross-section area, and total atheroma volume. This information is crucial for making decisions such as whether a stent is needed to restore blood flow in an artery and to determine the characteristics of the stent.

Manual segmentation of IVUS images is commonly performed by an observer. However, depending on the type of analysis, the number of frames that are needed to be segmented can range from a few frames to hundreds of frames. Therefore, the manual segmentation of those images may be excessively time-consuming. 
Moreover, the manual segmentation may suffer from inter- and intra-observer variability due to its high level of subjectivity. Studies have shown that there may exist large differences (up to 20\%) in the cross-sectional area of luminal segmentations provided by the same observer (Meier et al. (1997)). Thus, a robust automatic segmentation method for IVUS images is needed to overcome these limitations. However, automatic segmentation of IVUS images remains an open problem because of (i) the various artifacts that can appear in the IVUS images (e.g., shadows, speckle noise, side branches, and guidewire artifacts), and (ii) the variability on the gray level distribution for the different regions of interest depicted in the B-mode images which depends on the settings of the IVUS system (i.e., frequency of the transducer) and the image-generation parameters (i.e., gain, intensity compression, rejection, and gamma curves (Hiro et al. (1996))) that are subjectively adjusted by the interventionist during the acquisition.

In this paper, we present a probabilistic approach for the segmentation of the luminal border in IVUS images. The proposed method is based on the deformation of a curve which represents the lumen contour by minimizing a cost function that is formulated using a probabilistic approach in which the likelihoods of each pixel to belong to blood and non-blood are obtained by a Support Vector Machine (SVM) classifier. The SVM classifier is trained using a small number of samples from the lumen and non-lumen regions provided by the user on the first frame of the sequence to be segmented. The use of an SVM model to compute the likelihood of each pixel to belong to blood and non-blood regions makes the method applicable to IVUS images acquired with systems operating at different frequencies (i.e., 20 and $40 \mathrm{MHz}$ ) without the need of adjusting any parameter. Additionally, the proposed method includes a step in which possible changes in the gray level distributions of the regions of interest within a sequence are automatically detected, so that the likelihood computations can be progressively adapted. This step makes the method robust with respect to a possible change of gray level distributions within an IVUS sequence due to a change in the B-mode reconstruction parameters.

We have presented a preliminary version of this method in (Mendizabal-Ruiz et al. (2008)). The two major limitations of our previous method (MendizabalRuiz et al. (2008)) are the number of parameters to be tuned for the parameterization of the curve which uses a mixture of Gaussians, and the simple method for estimating the likelihood of the pixels to belong to lumen and non-lumen using gray-level histograms which limited the method to the segmentation of $20 \mathrm{MHz}$ IVUS sequences only. The method proposed here has three significant differences with respect to the work presented in (Mendizabal-Ruiz et al. (2008)). First, the 
lumen contour is parameterized using Fourier series, which is more suitable to the characteristics of the segmentation problem because it provides a smooth-periodic contour with a smaller number of parameters to be tuned when compared to the previous parameterization that used a periodic mixture of Gaussians. This parameterization also allows us to exclude the multi-scale approach proposed in the previous method. Second, the computation of the likelihood of the pixels to belong to lumen and non-lumen is based on the analysis of texture and the use of a SVM classifier. Third, an approach for the detection of possible incorrect segmentation based on the similarity of consecutive frames is proposed. The similarities with the previous method include the use of the same probabilistic cost function, the use of the same optimization method, and the need of user intervention for initialization.

The main contribution of our paper is a segmentation method that is robust with respect to the variability of gray level distributions of the regions of interest within the frames of an IVUS sequence and between different IVUS systems. Our method was evaluated on selected consecutive frames from twelve non-gated stationary IVUS sequences from two different IVUS systems using different frequencies, and the segmentation results were compared with other three existing segmentation methods.

The rest of the paper is organized as follows: Section 2 presents previous work in IVUS segmentation, while in Section 3 a detailed description of the proposed method is presented. Section 4 presents the results obtained with our method, and Sections 5 and 6 present our discussion and conclusions, respectively.

\section{Previous work}

Automated segmentation of IVUS sequences has been a topic of interest since the early 1990's. Many of the early approaches were based on the use of local properties of the image such as pixel intensity and gradient information (edges) combined with computational methods including graph search (Sonka et al. (1995), von Birgelen et al. (1996), Zhang et al. (1998)), active surfaces (Klingensmith et al. (2000)), active contours (Kovalski et al. (2000)), and neural networks (Plissiti et al. (2004)). In later approaches, segmentation was accomplished by the use of region and global information including texture (Mojsilovic et al. (1997)), gray level variances (Haas et al. (2000), Luo et al. (2003)) contrast between the regions (Hui-Zhu et al. (2002)), statistical properties of the image modeled by Rayleigh distributions using 2D (Haas et al. (2000), Brusseau et al. (2004)) and 3D information (Cardinal et al. (2006)), and by mathematical morphology techniques (dos 
Santos Filho et al. (2006)).

Recently, a shape-driven method for lumen and media-adventitia segmentation was introduced by Unal et al. (Unal et al. (2008)). In this work, the lumen and media-adventitia contours were constrained to a smooth, closed geometry. Then, a shape space was built using training data and principal component analysis (PCA). Finally, segmentation was performed on this shape space by the minimization of an energy function using nonparametric probability densities with global measurements. Taki et al. (Taki et al. (2008)) proposed a method for the delineation of the vessel borders. This method consisted of a preprocessing step followed by the geometric deformation of parametric models using edge information. Downe et al. (Downe et al. (2008)) introduced a method where principal component analysis was used for pre-processing, while active contour models were used to provide an initial segmentation for a 3D graph search method. Multilevel discrete wavelet frames decomposition was used by Papadogiorgaki et al. (Papadogiorgaki et al. (2008)) to generate texture information that was used along with the intensity information for contour initialization. Similarly, Katouzian et al. (Katouzian et al. (2008)) presented a method where texture information was extracted using a discrete wavelet packet transform. Then, pixels were classified as lumen or non-lumen using k-means clustering. Finally, the contour was parameterized using a spline curve. Our group presented a segmentation method based on the deformation of a lumen which represents the interface between lumen and wall (Mendizabal-Ruiz et al. (2008)). The segmentation was obtained by the minimization of a probabilistic cost function using gray level histograms to compute the likelihoods of the pixels to belong to class lumen and non-lumen. Bovenkamp et al. (Bovenkamp et al. (2009)) proposed an interactive method for IVUS segmentation based on the use of a multi agent image interpretation system which employs high-level knowledge-based control over low-level image segmentation algorithms. Ciompi et al. (Ciompi et al. (2009)) presented a method in which segmentation was tackled as a classification problem and solved using an error correcting output code technique. In that work, contextual information was exploited by means of conditional random fields computed from training data. Wennogle et. al (Wennogle and Hoff (2009)) proposed improvements over the method presented in (Cardinal et al. (2006)) which included a preprocessing step to remove motion artifacts, a new directional gradient velocity term, and a post-processing level-set method. Cardinal et al. (Cardinal et al. (2010)) presented a multiple interface 3D fast-marching method that was based on a combination of gray level probability density functions and the intensity gradient. The segmentation method included an interactive initialization procedure of the external vessel wall border. 
Zhua et al. (Zhu et al. (2011)) proposed a snake-based method for segmentation of IVUS images on which linear-filtered gradient vector flow which drives the deformation of a balloon snake. Moraes et al. (Moraes and Furuie (2011)) presented a method that relies on a binary morphological object reconstruction to segment the coronary wall in IVUS images. Balocco et al. (Balocco et al. (2011)) proposed a method based on the stabilization of the IVUS sequence and the subsequent registration of contiguous frames to generate a parametric image that distinguished the presence of tissues from blood. Segmentation was achieved by the classification of the pixels in the IVUS image using the growcut method. Katouzian et al. (Katouzian et al. (2012)) presented a 3D method which employs frequency-based harmonic information extracted by brushlet expansion. Then, the blood and non-blood regions of the IVUS image are detected by clustering the resulting brushlet coefficients. The detection of the lumen border is performed using surface function actives Duan et al. (2009) on the clustering result.

A common challenge for existing IVUS segmentation methods is that, besides the frequency of operation of the IVUS system, the gray level distribution of the different regions of interest depicted in the B-mode images depends on the reconstruction settings (e.g., time gain compensation (TGC), dynamic range compression, brightness, contrast and scaling) (Hiro et al. (1996)) (Fig. 2). These settings are subjectively selected by the interventionist (Mintz et al. (2001)), and may change from one intervention to the next, or even during the same acquisition.

In general, IVUS segmentation methods rely on the use of gray level intensity models that describe the regions of interest such as blood, plaque and vessel wall. However, these models and their parameters are computed using statistics over a determined training set of IVUS images. Therefore, these methods may require tuning or re-training in order to work appropriately on sequences containing IVUS images that are have different gray level distributions to those images in the training set because to the use of a different IVUS system or the use of different $\mathrm{B}$-mode reconstruction parameters.

\section{Material and methods}

Similarly to the work of Unal et al. (Unal et al. (2008)), we employ the Bmode polar IVUS image representation since this coordinate space makes the problem friendlier due to the 1D appearance of the lumen contour. In this representation, the horizontal axis corresponds to the angular position of the transducer, while the vertical axis corresponds to the radial penetration by the ultrasound beam. Therefore, the intensity value of a pixel $x=(r, \theta)$ can be defined 


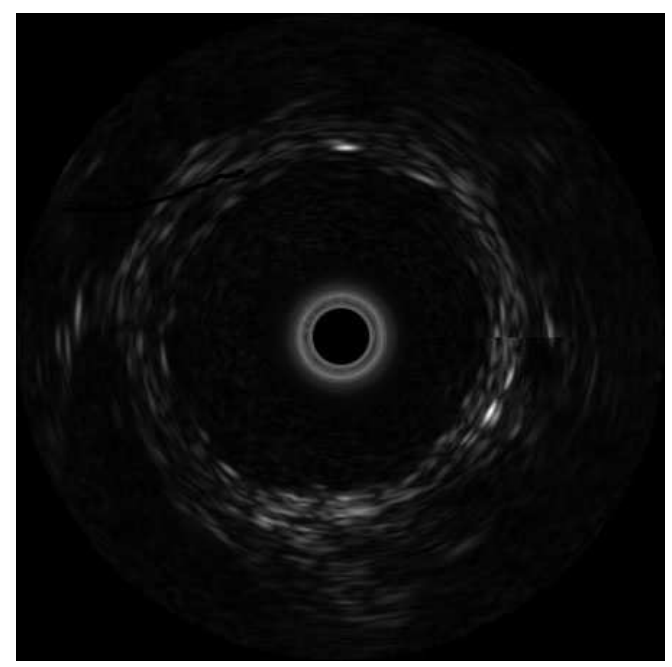

(a)

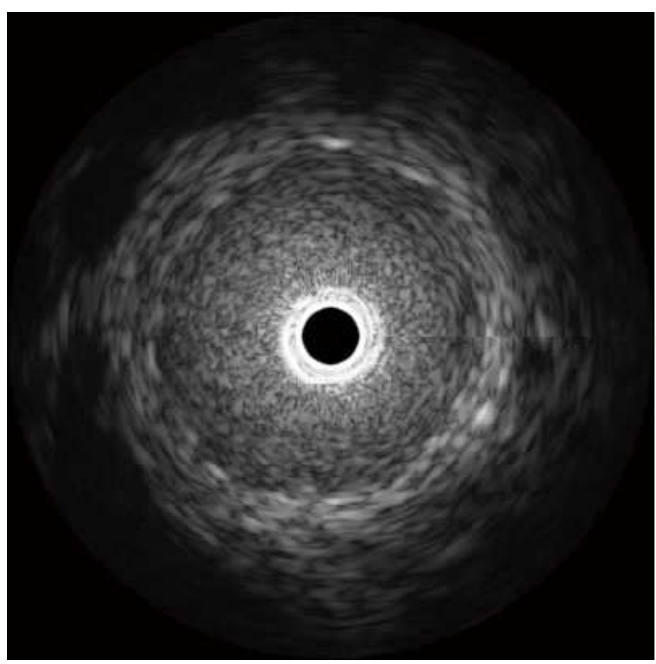

(b)

Figure 2: Example depicting a $40 \mathrm{MHz}$ IVUS frame in Cartesian B-mode representation using logarithmic dynamic range compression, (b) the same frame using linear dynamic range compression with a compression factor of 0.4 .

as $I(x)$ where $r$ and $\theta$ correspond to the radius and angle, respectively. We define the function $S$ that represents the interface between the lumen and the vessel wall (Fig. 3). This curve $S$ is parameterized by $\theta$ and $\vec{C}$ (i.e., $S(\theta, \vec{C})$ ).

\subsection{Lumen contour parameterization}

Manual annotations from observers define the lumen contour as a smooth curve. Moreover, a polar B-mode IVUS image is periodic with respect to the horizontal axis. Therefore, the smoothness and the periodicity of the contour are requirement for the parametric curve that defines the lumen/wall interface. In this work, we have chosen to parameterize this curve using Fourier series since it provides a periodic curve in which smoothness can be controlled by the number of coefficients $N_{k}$. The lumen contour is given by:

$$
S(\theta, \vec{C})=\frac{a_{0}}{2}+\sum_{k=1}^{N_{k}}\left[a_{k} \cos \left(\frac{k 2 \pi \theta}{N}\right)+b_{k} \sin \left(\frac{k 2 \pi \theta}{N}\right)\right],
$$




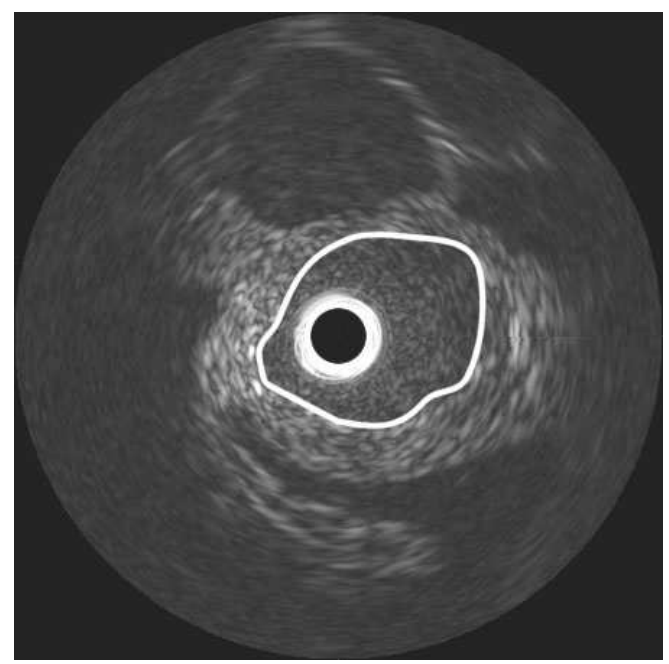

(a)

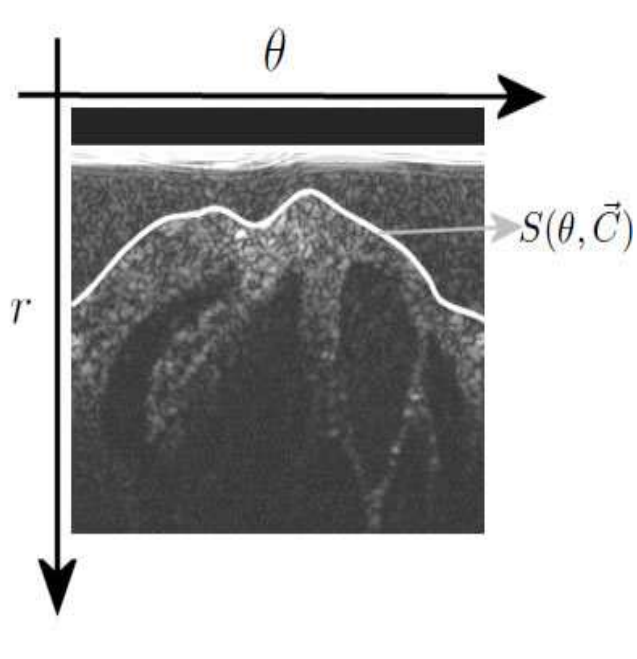

(b)

Figure 3: Depiction of the lumen contour in (a) Cartesian representation and (b) the polar B-mode representation.

where $N$ corresponds to the number of sampled angles (i.e., width of the polar image), and

$$
\vec{C}=\left[a_{0}, a_{1}, \ldots, a_{N_{k}}, b_{1}, \ldots, b_{N_{k}}\right]^{\top}
$$

are the Fourier coefficients that control the shape of the curve.

\subsection{Cost function for Probabilistic Segmentation}

In our method, the lumen segmentation problem consists of finding the optimum parameters $\vec{C}^{*}$ such that the curve $S\left(\theta, \vec{C}^{*}\right)$ corresponds to the interface between the lumen and the vessel wall. The formulation we introduce to solve this problem is inspired by the variational segmentation theory presented by Rivera $e t$ $a l$. (Rivera and Dalmau (2012)). This work propose to pose the $k$ classes segmentation problem as the computation of a probability field $P \in R^{k}$ with three main characteristics: (i) $P$ should be similar to a given likelihood field $\hat{V} \in R^{k}$, (ii) $P$ should be spatially smooth, and (iii) the entropy of $P$ should be controllable. Then, $P$ is computed by solving a problem of the form:

$$
\min _{p} U(P)=D(P, \hat{v})+\mu_{1} R_{1}(P)+\mu_{2} R_{2}(P),
$$


where $D$ is a term that promotes $P$ to be similar to $V$, and $\hat{v}_{k}(x)$ is the likelihood of the pixel $x$ for belonging to the class $k$. The regularization term $R_{1}$ controls the entropy of $P$ (desired to be as informative as possible) while the regularization term $R_{2}$ promotes $P$ to be spatially smooth. The relative contribution of the regularization terms to the total cost function $U(P)$ is controlled by the parameters $\mu_{1}$ and $\mu_{2}$.

The segmentation of the lumen region corresponds to a binary segmentation task (i.e., $P \in R^{2}$ ) on which the two possible classes for each pixel are lumen $(k=l)$ and non-lumen $(k=n)$. By definition, in the IVUS polar B-mode representation, the class of each pixel can be determined by evaluating the sign of the distance from the pixel to the curve that defines the lumen/wall interface $d(x, \vec{C})=S(\theta, \vec{C})-r$. If $d(x)>0$ the pixel belongs to lumen, otherwise the pixel belongs to non-lumen. Therefore, we define a parametric probability field $P$ for a polar B-mode image by using a sigmoid function which determine the probability of each pixel in the image to belong to the lumen $P_{l}(x)$ depending on its distance to the curve:

$$
P_{l}(x, \vec{C})=\frac{1}{1+e^{-\lambda d(x, \vec{C})}},
$$

where $\lambda$ is a parameter that controls the slope of the sigmoid.

Using this formulation, pixels far above the contour will have a higher probability of belonging to the lumen, while the pixels far below the contour will have probability close to zero. For the pixels near the contour, depending on the value of $\lambda$, the probability of belonging to lumen will be close to 0.5 (Fig. 4). The probability of a pixel to belong to non-lumen is given by: $\left(1-P_{l}(x)\right)$.

In our problem, the entropy is related to the sharpness of the transition between the two classes (from $P \approx 1$ to $P \approx 0$ ). This transition is controlled by the parameter of the sigmoid $\lambda$ resulting in a similar effect that the entropy control term $R_{1}$ of Eq. (2). Similarly, in our case the effect of the regularization term $R_{2}$ of Eq. (2) which promotes the spatial smoothness of $P$, is controlled by the number of Fourier coefficients $N_{k}$ corresponding to the parametric curve $S(\theta, \vec{C})$.

In (Rivera and Dalmau (2012)) several choices for the term $D$ are discussed. In this work, we chose to use the Kerridge's inaccuracy measure:

$$
D(P, V)=-\sum_{k} \sum_{x} P_{k}(x) \log \hat{v}_{k}(x) .
$$

which is a generalization of the Shannon entropy used to measure the inaccuracy of the assertion of a probability estimation (Kerridge (1961)). 


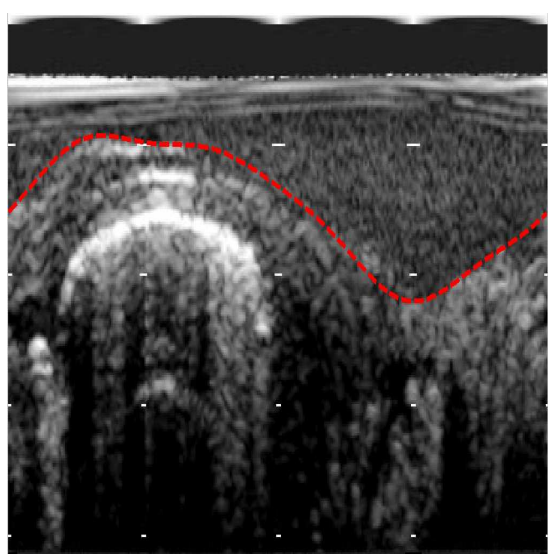

(a)

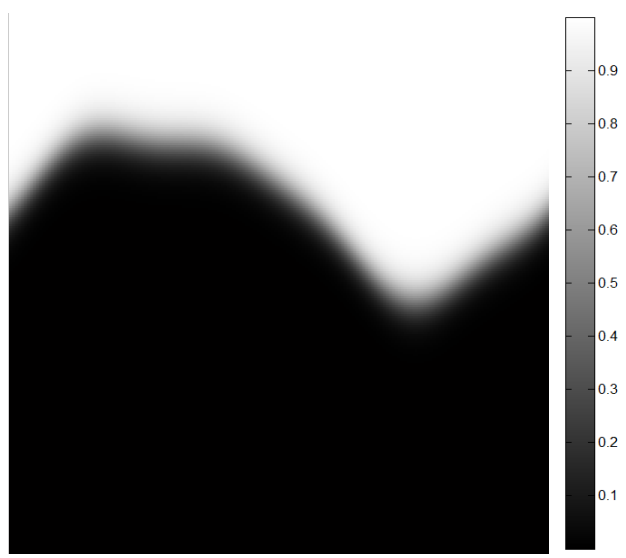

(b)

Figure 4: (a) Example depicting the curve corresponding to the lumen/wall interface, and (b) the parametric probability field defined by the curve using $\lambda=0.8$.

Let $\hat{v}_{l}(x)$ and $\hat{v}_{n}(x)$ be the likelihood of the pixel $x=(r, \theta)$ to belong to the lumen and non-lumen, respectively. Then, the lumen segmentation problem is posed as the minimization of the cost function:

$$
U(\vec{C})=-\sum_{x} P_{l}(x, \vec{C})\left[\log \left(\hat{v}_{l}(x)\right)\right]+\left[1-P_{l}(x, \vec{C})\right]\left[\log \left(\hat{v}_{n}(x)\right)\right] .
$$

The details regarding the computation of these likelihoods are provided in Sec. 3.4 .

Note that the proposed cost function (Eq. 5) is consistent with the spirit of the probabilistic framework proposed by Rivera et al. since (i) the probabilistic segmentation $P$ is attached by the Kerridge information measure to the likelihood measure field $V$, (ii) the smoothness of $P$ is controlled by the number of coefficients that define the parametric boundary curve, and (iii) the slope of $P$ at the class boundary (i.e., entropy) is controlled by the parameter $\lambda$ of the sigmoid (Eq. (3)).

\subsection{Optimization}

To deform the lumen contour until it reaches the best segmentation, it is necessary to find the values of $\vec{C}^{*}$ that minimize the cost function of Eq. (5). In this work, we propose to use a line search method for this minimization. In a line 
search method, the optimal value $\vec{C}^{*}$ is computed iteratively. At every iteration $k$, the new value for $\vec{C}_{k+1}$ is computed by:

$$
\vec{C}_{k+1}=\vec{C}_{k}+\alpha_{k} \vec{P}_{k}
$$

where $\alpha_{k}$ is the step length at the iteration $k$ and $\vec{P}_{k}$ is a descent or search direction. A popular way to find an adequate value of $\alpha_{k}$ is by searching the one that satisfies the Wolfe conditions (Nocedal and Wright (1999), Chapter 3):

$$
\begin{gathered}
U\left(\vec{C}_{k}+\alpha_{k} \vec{P}_{k}\right) \leq U\left(\vec{C}_{k}\right)+u_{1} \alpha_{k} \nabla U\left(\vec{C}_{k}\right)^{\top} \vec{P}_{k} \\
\nabla U\left(\vec{C}_{k}+\alpha_{k} \vec{P}_{k}\right)^{\top} \vec{P}_{k} \geq u_{2} \nabla U\left(\vec{C}_{k}\right)^{\top} \vec{P}_{k} .
\end{gathered}
$$

For selecting the descent direction $\vec{P}_{k}$ at each iteration $k$ we have several options. We will discuss two options below.

The steepest descent method: One of the simplest ways to approximate the solution of optimization problems is by using the steepest descent method (Nocedal and Wright (1999)). For an objective function $U(\vec{C})$, the steepest descent method is a line search method that iteratively moves along a search direction given by:

$$
\overrightarrow{P_{k}^{G}}=-\frac{\nabla U\left(\vec{C}_{k}\right)}{\left|\nabla U\left(\vec{C}_{k}\right)\right|}
$$

Although this method is robust and guarantees convergence to a local minimal, the main disadvantage is that this method may take a large number of iterations (i.e., time) to converge to the solution.

Broyden-Fletcher-Goldfarb-Shanno (BFGS) method: The BFGS method (Nocedal and Wright (1999)) converges to a solution faster when compared to the steepest descent method since it incorporates first and second order derivatives to find the point at which the gradient of the function is equal to zero. The BFGS algorithm is efficient since, instead of computing the inverse of the Hessian from scratch at every iteration, it is updated in a simple manner by taking into account the curvature measured in the most recent step. In this method, the search direction at each iteration $k$ is given by:

$$
\vec{P}_{k}^{B}=-H_{k} \nabla U\left(\vec{C}_{k}\right),
$$

where the matrix $H_{k}$ is the inverse of a positive definite approximation of the Hessian of the cost function at each iteration computed as:

$$
H_{k+1}=\left(I-\rho_{k} \vec{s}_{k} \vec{y}_{k}^{\top}\right) H_{k}\left(I-\rho_{k} \vec{y}_{k} \vec{s}_{k}^{\top}\right)+\rho_{k}{\overrightarrow{s_{k}}}_{\vec{s}_{k}^{\top}}^{\top}
$$


with

$$
\rho_{k}=\frac{1}{\vec{y}_{k}^{\top} \overrightarrow{s_{k}}}
$$

were $\overrightarrow{s_{k}}=\vec{C}_{k+1}-\vec{C}_{k}$ and $\vec{y}_{k}=\nabla U\left(\vec{C}_{k+1}\right)-\nabla U\left(\vec{C}_{k}\right)$ which must satisfy the curvature condition

$$
\vec{s}_{k}^{\top} \vec{y}_{k}>0
$$

In our problem, depending on the IVUS image to segment, the cost functions $U(\vec{C})$ may have many local minima mostly due to the similarity between the gray levels distributions of lumen and other regions of the vessel. Therefore, solving the cost function with the BFGS method could lead us to an incorrect segmentation if the descent step is too large in a given iteration. Note that the steepest descent method is robust but slow, while BFGS is fast but may converge into an incorrect solution (Fig. 5). Therefore, we propose a strategy for combining the strengths of the two methods which uses a linear combination of the descent directions from steepest descent $\left(\vec{P}^{G}\right)$ and BFGS $\left(\vec{P}^{G}\right)$ methods to compute a new descent direction. The linear combination of these methods is denoted as $\left(\vec{P}^{G B}\right)$ and therefore, we refer to this strategy as GB optimization .

GB optimization: An advantage of the BFGS algorithm over other Newtonbased methods such as Levenberg-Marquart (LM) is the direct computation of the inverse of the Quasi-Hessian $H$. However, the reason we chose BFGS over LM is because the information obtained from the curvature condition Eq. (13).

When this condition is satisfied, the curvature of the function becomes more positive as the descent approaches to a minimum. However, if the curvature condition is not satisfied, a better descent direction is the negative gradient (i.e., steepest descent direction). Additionally, note that for small values of the product $\vec{s}_{k}^{\top} \vec{y}_{k}$, the computation of the update formula for the Hessian, or its inverse, is non-defined (the function is not locally convex). Then, by design there is more confidence in the BFGS descent direction if the value of $\rho$ (Eq. (12)) is large and positive. On the other hand, for $\rho \approx 0$, the confidence in $\vec{P}_{k}^{B}$ is smaller and we prefer the steepest descent direction $\vec{P}_{k}^{G}$. Based on this analysis, we propose to compute the descent direction as a convex linear combination of both descent directions (Mendizabal-Ruiz et al. (2008)):

$$
\vec{P}_{k+1}^{G B}=\left[\psi\left(\rho_{k}\right) \vec{P}_{k}^{B}+\left(1-\psi\left(\rho_{k}\right)\right) \vec{P}_{k}^{G}\right] .
$$

where the function that controls the contribution of each descent direction 
$\psi(\rho)$ is defined as:

$$
\psi(\rho)= \begin{cases}0 & \text { if } \rho<0 \\ \frac{\rho^{2}}{K+\rho^{2}} & \text { otherwise } .\end{cases}
$$

Algorithm 1 describes the proposed optimization method.

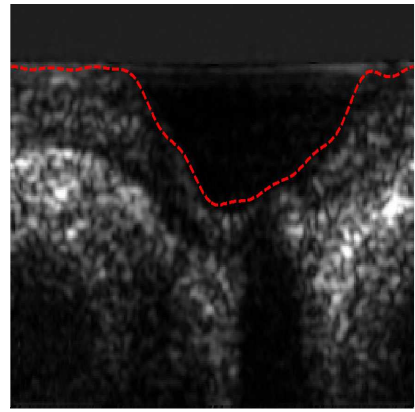

(a)

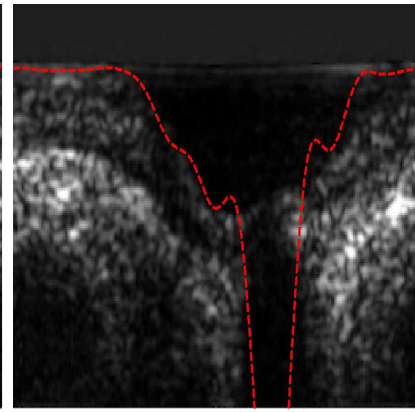

(b)

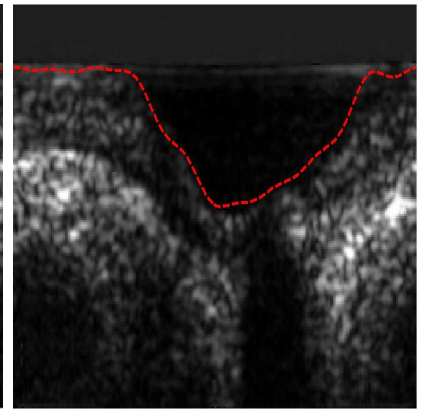

(c)

Figure 5: Examples of a segmentation result on a $20 \mathrm{MHz}$ IVUS frame using (a) steepest descent optimization (72 iterations), (b) BFGS optimization (38 iterations), and (c) GB optimization (26 iterations).

\subsection{Likelihood estimates}

\subsubsection{Texture descriptors}

The intensity of the blood speckle increases exponentially with the increase of the frequency of the transducer (Mintz et al. (2001)). For example, in $20 \mathrm{MHz}$ images the gray level distribution of the pixels in the lumen region appears relatively dark when compared to $40 \mathrm{MHz}$ images which depict some texture due to the speckle. Therefore, in this work, we propose to incorporate texture information into the terms corresponding to the likelihoods of the pixels to belong to lumen and non-lumen. Laws' texture measures are a well known, widely used class of textural image descriptors and enjoy continued use today mostly due to their relatively simple implementation, and the fact that they mimic the behavior of more advanced methods (Lee and Schenk (1992)). Laws' texture features are generated from an image by first convolving the image with a number of specialized kernels. These kernels are in the majority of cases $5 \times 5$ (level 5), though others were proposed which do not currently find widespread use. The level 5 kernels are produced by taking the outer product of all the combinations of the 


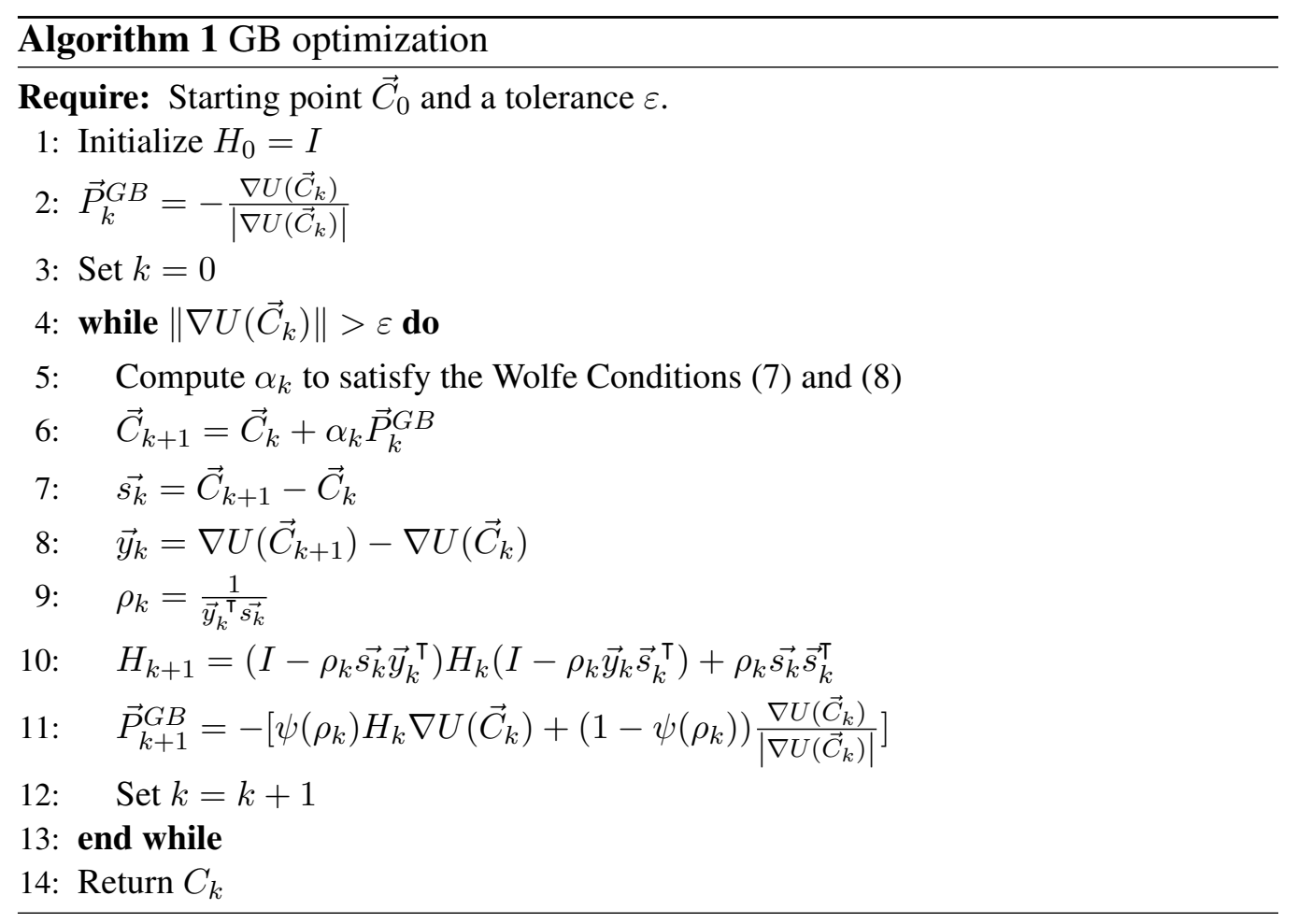


Table 1: One-dimensional convolution kernels for level 5.

\begin{tabular}{|c|ccccccc|}
\hline Name & \multicolumn{7}{|c|}{ Kernel } \\
\hline Levels (L5) & {[} & 1 & 4 & 6 & 4 & 1 & ] \\
\hline Edges (E5) & {[} & -1 & -2 & 0 & 2 & 1 & ] \\
\hline Spots (S5) & {[} & -1 & 0 & 2 & 0 & -1 & ] \\
\hline Waves (W5) & {[} & -1 & 2 & 0 & -2 & 1 & ] \\
\hline Ripples (R5) & {[} & 1 & -4 & 6 & -4 & 1 & ] \\
\hline
\end{tabular}

basis one-dimensional level 5 convolution kernels (Table 1) (Laws (1980)). The 2D kernels are assigned a string mnemonic $k_{L} k_{E}$ (e.g., Kernel L5E5 is generated by convolving vertical kernel L5 by horizontal kernel E5). A total of 25 images are formed by convolving the original $2 \mathrm{D}$ images with the convolution kernels.

After convolving the polar B-mode image with these kernels, it is necessary to convert each of the resulting 25 images $L_{l}$ into a measure of texture energy $E_{l}$. This is accomplished by a windowing operation in which the value of every pixel $x$ is replaced by the absolute value of the sum of the pixel values in a $5 \times 5$ window $W_{x}$ centered in the pixel $x$

$$
E_{l}(x)=\sum_{y \in W_{x}}\left|L_{l}(y)\right| .
$$

These texture energies are used to form a 25-dimensional feature vector $\vec{E}_{x}=\left[E_{1}, E_{2}, \ldots, E_{25}\right]$ that will characterize the class of each pixel $x$.

\subsubsection{Support vector machines (SVM) classifier}

SVM is a class of binary supervised learning methods that are commonly used for classification and regression. SVMs process the data by mapping all samples features into a high dimensional space defined by a kernel. In this high dimensional space, the data may be linearly separated by a single boundary defined by a hyperplane where support is given by selected samples in the data (support vectors). To increase the probability of a correct classification it is desirable to have the decision boundary at a maximum possible distance from the samples. The distance between the support vectors and the decision boundary is called the margin. The goal of SVM, is to find a separating hyperplane that would result in the largest margin. Once the optimal hyperplane function is computed, any new sample can be classified by mapping its features into the same space defined by the kernel and 
then evaluating in the discriminant function ${ }^{2}$. In this work, we chose to employ the radial basis function (RBF) kernel (i.e., $k\left(\vec{E}_{i}, \vec{E}_{j}\right)=\exp \left(-\gamma\left\|\vec{E}_{i}-\vec{E}_{j}\right\|^{2}\right), \gamma>0$, for a pair of feature vectors $\vec{E}_{i}$ and $\vec{E}_{j}$ ). One of the motivations to use the RBF kernel, is that it requires only two parameters to be tuned ( $c$ which corresponds to the penalty for errors in the classification, and $\gamma$ corresponds to the width of the RBF kernel) as compared with other kernels (e.g., polynomial) (Hsu et al. (2003)). A common strategy for finding the values for these hyper parameters is by using cross-validation and grid-search (Hsu et al. (2003)). In addition to the class of a given sample, it is possible to compute the posterior probability estimate of the SVM prediction $P\left(k \mid \vec{E}_{x}\right)$ (Wu et al. (2004)). In this work, we propose to use $P\left(k \mid \vec{E}_{x}\right)$ as the learned likelihood for lumen and non-lumen $\hat{v}_{l}(x)$ and $\hat{v}_{n}(x)$, respectively.

\subsubsection{Computation of likelihoods}

In general, we can assume that the gray level distributions and texture of the lumen and non-lumen regions remain similar within the frames belonging to the same sequence. We part from this assumption to compute the likelihoods based on the use of a SVM classifier that is trained using texture features of a small number of samples from the lumen and non-lumen regions given by the user in the first frame of the sequence to be segmented. However, although the assumption of similarity of gray level distributions within the frames of the same sequence is valid most of the time, minor differences in the gray level distribution of the lumen region on the frames can be expected since the gray level distribution of speckle is also related to blood velocity, where low-velocity blood produces coarser, brighter speckle and higher velocities produce finer, lower-intensity speckle (Nissen and Yock (2001)). Moreover, possible changes in the B-mode reconstruction parameters during the acquisition may result in large differences between the gray level distributions of frames within the same sequence. If any of these cases occur, it may be possible that the original SVM model that was generated using the samples from the first frame is not reliable for computing the likelihoods for those frames with different gray level distributions. One option to overcome this limitation is to train the SVM using samples from all the frames in the sequence which depict significant changes in the gray level intensities of the regions of interest. However, it is the intention of this work to require the less user intervention as possible. Therefore, we propose to detect the possible changes on the gray level

\footnotetext{
${ }^{2}$ For more information regarding the theory of SVM we refer the reader to (Burges (1998)).
} 
intensities during the sequence and then adjusting the SVM model to account for this possible changes. Then, the process of estimating the likelihoods can be divided into three steps: training, deployment, and model adjustment.

Training: In the training step, we require the user to select samples of lumen and non-lumen regions ( $R_{l}$ and $R_{n}$, respectively) from the first frame $f_{1}$ of the sequence to be segmented (Fig. 6). We compute the texture energies $L(x) \forall x \in\left\{R_{l}, R_{n}\right\}$ in the IVUS polar B-mode image using the Law's filters as described previously. A feature vector $\vec{E}_{x} \in R^{25}$ containing the computed texture energies for each pixel corresponding to $R_{l}$ and $R_{n}$ is associated to its corresponding class $k(x) \in\{l, n\}$. A training set $T_{1}$ is then generated using the feature vectors and the classes of each pixel. The optimal hyper parameters of the SVM model using the RBF kernel $c$ and $\gamma$ are found using a grid search with 5-fold cross-validation (Hsu et al. (2003)). Next, a SVM model $\Pi_{1}$ is computed using the training set $T_{1}$ and the optimal $c$ and $\gamma$.

Deployment: For each of the frames to be segmented, we compute a vector $\vec{E}_{x}$ for every pixel in the frame $f_{i}$ and we obtain its class $k(x)$ using $\Pi_{1}$. The posterior probabilities of the classification result for each pixel are then used as the learned likelihoods $\hat{v}_{l}(x)=P\left(l \mid \vec{E}_{x}\right)$ and $\hat{v}_{n}(x)=P\left(n \mid \vec{E}_{x}\right)$.

Ringdown artifact: Cartesian B-mode frames depict a dark circle in the middle of the image. This circles are generated by the IVUS system and corresponds to the IVUS catheter for which there is no available information. Ring-down artifacts are produced by acoustic oscillations in the transducer which are usually observed as bright halos of variable thickness surrounding the catheter creating a zone of uncertainty adjacent to the transducer surface (Mintz et al. (2001)). These artifacts are commonly present in the region corresponding to the lumen and may interfere with the computation of the likelihoods for the lumen. However, our observations in numerous IVUS sequences indicate that the width of this halo remains relatively constant within a sequence. Therefore, since the region occupied by the ring down artifact correspond to lumen we set the likelihoods of those pixels to belong to lumen to one. We require the user intervention to provide the radial location $\chi$ of the outer-most trace of the ringdown artifact in the first polar B-mode frame of the sequence to be segmented. Then, we set the likelihoods for the region corresponding to the catheter and the ring down artifact as $\hat{v}_{l}(x)=1 \forall x \mid r \leq \chi$ and $\hat{v}_{n}(x)=0 \forall x \mid r \leq \chi$. Figure 6 depicts examples of the likelihood computed for a frame as computed with the SVM model.

Model adjustment: Considering the speed of the IVUS images acquisition (30 frames $/ \mathrm{s})$ and the typical pullback speed using motorized device $(0.5 \mathrm{~mm} / \mathrm{s})$, we 
can assume that the distance between the cross sections of the vessel depicted by two consecutive frames is approximately $16 \mu \mathrm{m}$. Then, in general we assume the shape of the lumen contour of two consecutive frames $i$ and $i+1$ is very similar and therefore the maximum distance between the lumen contour of these consecutive frames is constrained to $w_{b}$ (an experiment for validating this assumption is presented in Sec. 5.1.2). We propose to use this information to assess the reliability of the SVM model trained with the samples acquired from the first frame of the sequence to detect the possible changes in the gray level intensities of the lumen and non-lumen regions by assessing its accuracy on the classification of the regions defined by the curve $S_{i}\left(r, \theta, \vec{C}_{i}\right)$ on the next consecutive frame $S_{i+1}$ in the following way.

Consider the segmentation result $S_{i}$ for a frame $i$. If we place the curve $S_{i}$ on the next consecutive frame $i+1$, we can assume that most of the pixels above and below the curve will effectively belong to the corresponding classes with exception of those pixels that are closer to the curve. We define a distance $w_{b}$ around this curve and we assume that the pixels with $d\left(x, \vec{C}_{i}\right) \geq w_{b}$ correspond to lumen while those pixels with $-w_{n} \leq d\left(x, \vec{C}_{i}\right) \leq-w_{b}$ correspond to non-lumen. We introduce $w_{n}$ as a limit below the curve is because we are only interested in the non-lumen regions more proximal to the lumen in order to discard other outer regions such as adventitia or outer adventitia. This information is used to create a class mask (Fig. 7), and we use the classes of these pixels as ground truth for assessing the classification result of the texture features of frame $i+1$ for the pixels on the mask. If the accuracy $A_{1}$ of the classification is above a given threshold (i.e., $A_{1} \geq \tau_{1}$ ), we assume that we can rely on the classification result and therefore we use the classes and probabilities of the prediction given by $\Pi_{1}$ as the likelihoods for the segmentation of this frame. However, if the $A_{1}<\tau_{1}$ this may be an indication of a change on the gray level distributions of the regions of interest or a large change on the shape of the lumen contours from frame $i$ to frame $i+1$. Then, we perform the following steps: first we assume that the reason for the low accuracy obtained is due to a change in the gray level distribution. Then, we build a new training set $T_{2}$ consisting of randomly selected samples from the original training set $T_{1}$ and from the blood and non blood regions on the frame $i+1$ defined by the class mask. Then, we train a new SVM model $\Pi_{2}$ in using $T_{2}$. The accuracy $A_{2}$ of $\Pi_{2}$ is evaluated by classifying all the pixels within the class mask. If $A_{2}$ improves with respect to $A_{1}$ (i.e., $A_{2} \geq A_{1}$ ), we consider that the new model is more reliable and therefore it is used to estimate the likelihoods of the subsequent frames. However, if $A_{2}<A_{1}$, we assume that the low accuracy is 
the result of a large change on the shape of the lumen occurred and therefore the previous SVM model $\Pi_{1}$ is maintained to compute the likelihoods of the pixels of that frame and the subsequent frames. This process is repeated for each frame in the sequence leading to a progressively adapting blood and non-blood model.

After the likelihoods for each pixel in the image are defined. The segmentation of the frame is performed by the minimization of Eq. 5. Algorithm 2 presents the steps of the proposed B-mode approach.

\section{Results}

The similarity between the lumen shape of consecutive frames, the sensitivity of our method with respect to its parameters $\lambda$ and $N_{k}$, and the overall performance of our method in the segmentation of IVUS B-mode Cartesian images, was evaluated on twelve non-gated stationary IVUS sequences from two IVUS systems using different frequencies. Six sequences were acquired from human coronary arteries from different patients using a Volcano system with a $20 \mathrm{MHz}$ Eagle Eye catheter, and the remaining six were acquired from Rabbit's aortas using a Boston Scientific Galaxy 2 system with a $40 \mathrm{MHz}$ Atlantis SR Catheter. From each sequence a number of consecutive frames corresponding to regions of interest within the artery (different degrees of stenosis) were selected and manually segmented by human observers ( 585 frames in total). To compare the performance our method (MRK) with other existing segmentation methods, we asked three research groups to perform segmentation in the selected data sets using their segmentation methods. The original IVUS data was provided to each author and they provide us with the segmentation results. The methods used for comparison correspond to the methods presented by Unal et al. (UNL) (Unal et al. (2008)), Papadogiorgaki et al. (PAP) (Papadogiorgaki et al. (2008)), and Katouzian et al. (KAT) (Katouzian et al. (2008)). The details of each sequence and the number of segmented frames provided by each group are listed in Table $2^{3}$. The method proposed in this paper was implemented using MATLAB ${ }^{\circledR}$ employing the SVM implementation provided in the libSVM library (Chang and Lin (2011)).

\subsection{Parameter selection}

We define the starting point for the first frame to be segmented by setting the offset coefficient $a_{0}$ to be equal to the mean of the radial coordinates of the blood

\footnotetext{
${ }^{3}$ The missing segmentation results were not provided by the corresponding authors.
} 


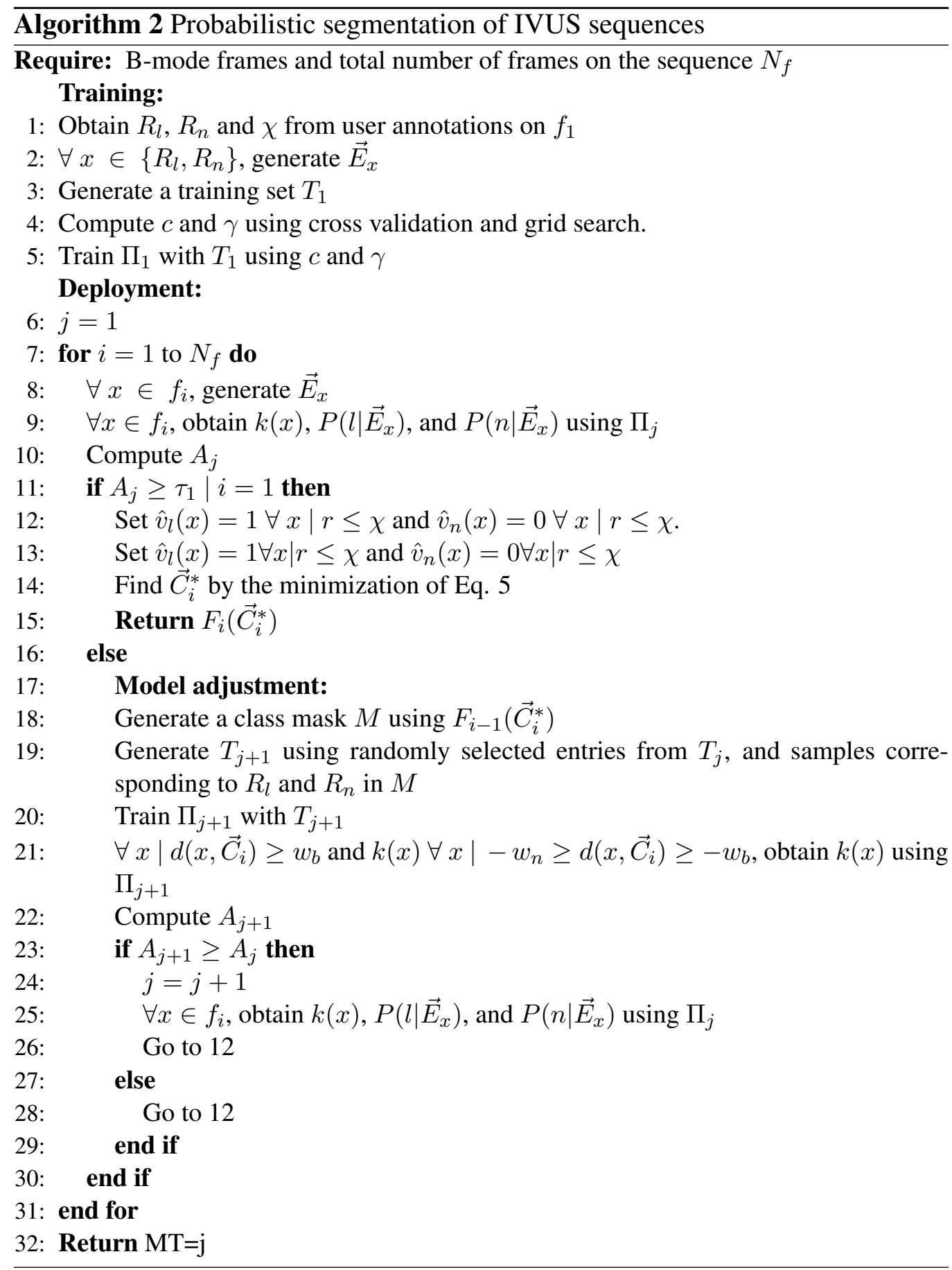


Table 2: Information about the IVUS sequences and the number of segmented frames using our method (MRK), Unal's method (UNL), Papadogiorgaki's method (PAP), and Katouzian's method (KAT).

\begin{tabular}{|c|c|c|c||c|c|c|c|}
\hline \multicolumn{4}{|c||}{ Sequence information } & \multicolumn{4}{c|}{ Number of segmented frames } \\
\hline ID & IVUS system & Frequency & Subject & MRK & UNL & PAP & KAT \\
\hline 1 & Volcano & $20 \mathrm{MHz}$ & Human & 50 & 50 & 50 & 48 \\
\hline 2 & Volcano & $20 \mathrm{MHz}$ & Human & 50 & 50 & 50 & 0 \\
\hline 3 & Volcano & $20 \mathrm{MHz}$ & Human & 50 & 50 & 50 & 0 \\
\hline 4 & Volcano & $20 \mathrm{MHz}$ & Human & 50 & 50 & 50 & 0 \\
\hline 5 & Volcano & $20 \mathrm{MHz}$ & Human & 50 & 50 & 50 & 0 \\
\hline 6 & Volcano & $20 \mathrm{MHz}$ & Human & 50 & 50 & 50 & 0 \\
\hline 7 & Boston Scientific & $40 \mathrm{MHz}$ & Rabbit & 50 & 50 & 50 & 48 \\
\hline 8 & Boston Scientific & $40 \mathrm{MHz}$ & Rabbit & 50 & 50 & 50 & 48 \\
\hline 9 & Boston Scientific & $40 \mathrm{MHz}$ & Rabbit & 50 & 50 & 50 & 48 \\
\hline 10 & Boston Scientific & $40 \mathrm{MHz}$ & Rabbit & 50 & 50 & 50 & 48 \\
\hline 11 & Boston Scientific & $40 \mathrm{MHz}$ & Rabbit & 50 & 50 & 50 & 48 \\
\hline 12 & Boston Scientific & $40 \mathrm{MHz}$ & Rabbit & 35 & 35 & 35 & 32 \\
\hline
\end{tabular}

samples provided by the user. The values for the rest of the coefficients set to $\left\{a_{i}, b_{i}\right\}=0.1 \forall i>0$. The segmentation method parameters were set to $\lambda=0.4$ and $N_{k}=5$ since this values report a good tradeoff between the average frame segmentation time and similarity with respect to the manual segmentations (Sec. 5.1.1). Considering that for more than $90 \%$ of the frames the largest radial difference is less than 20 pixels (Sec. 5.1.2) we set $\tau_{1}=80$ and $w_{b}=20$. We empirically set $w_{n}=50$ in order to have a band of distance of 30 pixels corresponding to non-blood region.

\subsection{IVUS data experiments}

The performance of our method was evaluated by comparing our segmentation results with the manual segmentation of the same frames by one observer. Specifically, we computed the Dice $D$ similarity and the Jaccard index between the areas reported by the different segmentations. In addition, we computed the Hausdorff distances between the corresponding lumen curves. The agreement between the areas corresponding to lumen according to each segmentation was evaluated using linear regression, coefficient of determination and Bland-Altman analysis (Bland and Altman (1986)). The number of times the model was computed along with its total time, and the average time for segmenting each frame are listed in Table 3. The mean Dice similarity and standard deviation results for the comparison of both manual segmentation and each automatic segmentation method are depicted 
as a box plot in Fig 8. The segmentation results corresponding to each of the sequences for the comparison of both observers, and the MRK, UNL, PAP, and KAT methods and both observers, are listed in Tables 4, 5, 6, 7, 8, respectively.

Note that the results obtained with the MRK method are closer and more consistent with the manual segmentation when compared with the other automatic methods. Figure 9 depict the linear regression and Bland-Altman plots for the comparison of the lumen areas corresponding to the segmentations of Observer 1 and Observer 2. Figures 10 to 17, depicts the linear regression and Bland-Altman plots for the comparison of the lumen areas corresponding to the segmentation of both observers segmentation with the segmentation obtained with the MRK, UNL, PAP, and KAT methods respectively. Note that the linear regression results indicate a good agreement between the luminal area detected by the MRK method with the luminal area defined by the observers and less dispersion as compared by the area detected by the other methods. Moreover, the MRK method reports a higher coefficient of determination when compared with the other methods. From the Bland-Altman plots it can be observed that MRK method perform with a low mean bias and less dispersion when compared with the other methods. Figures 18 and 19 depict examples of the segmentation results for each method along with the segmentation by the Observer 1 .

To determine the statistical significance of the performance of the MRK method compared with the other three methods, we perform three paired Wilcoxon signed rank test analysis (MRK vs. UNL, MRK vs. PAP, and MRK vs. KAT). For this analysis, we perform block-sampling from the set of all Dice coefficient results for the comparison of each method with Observer 1 (one frame every five). Then, runs test analysis was performed in order to verify independence within the samples as required for the signed rank test. We perform the Wilcoxon signed rank test using the following hypothesis:

1. Null hypothesis: the median of the MRK method is equal to the median of the compared method (UNL, PAP or KAT).

2. Alternative hypothesis: the difference of the medians of the MRK and the compared method is larger than 0 .

In addition, we performed a Levene's test to assess the difference of variances reported by each method using the following hypothesis:

1. Null hypothesis: the variance of the MRK method is equal to the variance of the compared method (UNL, PAP or KAT).

2. Alternative hypothesis: the variance of the MRK method is different from the variance of the compared method (UNL, PAP or KAT). 
Table 3: Average segmentation time per frame (ST), number of times that the SVM model was trained (MT), total SVM model training time (TT), and total segmentation time (TS) for each sequence.

\begin{tabular}{|c|r|c|r|r|}
\hline ID & ST $(s)$ & MT & TT $(s)$ & TS $(s)$ \\
\hline 1 & 3.04 & 1 & 3.38 & 155.30 \\
\hline 2 & 3.43 & 1 & 4.13 & 175.63 \\
\hline 3 & 3.42 & 1 & 3.21 & 174.21 \\
\hline 4 & 3.14 & 1 & 7.23 & 164.23 \\
\hline 5 & 2.71 & 1 & 2.38 & 137.88 \\
\hline 6 & 4.21 & 1 & 9.45 & 219.95 \\
\hline 7 & 5.11 & 1 & 8.52 & 264.02 \\
\hline 8 & 7.08 & 2 & 33.2 & 387.20 \\
\hline 9 & 5.75 & 1 & 9.54 & 297.04 \\
\hline 10 & 5.15 & 2 & 24.31 & 281.81 \\
\hline 11 & 5.16 & 1 & 13.12 & 271.12 \\
\hline 12 & 4.55 & 1 & 13.02 & 172.27 \\
\hline
\end{tabular}

For each test we employ a confidence level of $\alpha=0.05$ employing the Bonferroni correction $\hat{\alpha}=\frac{\alpha}{m}$, where $m=3$ is the number of paired test.

The results for the signed rank test indicate that the null hypothesis is rejected with $\mathbf{p}$ values of $2.19 \times 10^{-9}$ for MRK vs. KAT, $4.15 \times 10^{-11}$ for MRK vs. PAP, and $4.8 \times 10^{-7}$ for MRK vs. KAT. Similarly, the results for the Levene's test indicate that the null hypothesis is rejected with $\mathbf{p}$ values $\approx 0$ for all the comparison cases. 
Table 4: Mean Dice similarity, Jaccard index, and Hausdorff distance and standard deviations (std) for the comparison of the segmentation results of Observer $1(\mathrm{O} 1)$ and Observer $2(\mathrm{O} 2)$. The mean is denoted by $\mu$ and the for the standard deviation by $\sigma$.

\begin{tabular}{|r|r|r|r|r|r|r|}
\hline & \multicolumn{2}{|c|}{ Dice } & \multicolumn{2}{c|}{ Jaccard } & \multicolumn{2}{c|}{ Hausdorff } \\
\hline ID & $\mu(\%)$ & $\sigma(\%)$ & $\mu(\%)$ & $\sigma(\%)$ & $\mu(\%)$ & $\sigma(\%)$ \\
\hline 1 & 94.17 & 2.42 & 89.07 & 4.24 & 8.51 & 3.43 \\
\hline 2 & 92.92 & 1.86 & 86.84 & 3.23 & 12.88 & 4.11 \\
\hline 3 & 90.10 & 3.11 & 82.13 & 5.15 & 15.69 & 4.59 \\
\hline 4 & 92.73 & 2.96 & 86.58 & 5.04 & 14.35 & 4.24 \\
\hline 5 & 92.53 & 3.06 & 86.24 & 5.23 & 14.05 & 5.73 \\
\hline 6 & 92.53 & 1.79 & 86.15 & 3.06 & 25.09 & 6.51 \\
\hline 7 & 96.58 & 1.11 & 93.40 & 2.06 & 7.05 & 2.23 \\
\hline 8 & 97.67 & 0.51 & 95.45 & 0.98 & 7.56 & 2.12 \\
\hline 9 & 96.45 & 1.04 & 93.16 & 1.92 & 12.97 & 4.94 \\
\hline 10 & 96.35 & 0.96 & 92.97 & 1.78 & 10.54 & 3.68 \\
\hline 11 & 97.84 & 0.52 & 95.77 & 1.00 & 5.57 & 1.34 \\
\hline 12 & 96.46 & 1.03 & 93.18 & 1.92 & 11.99 & 3.59 \\
\hline Average & $\mathbf{9 4 . 6 9}$ & $\mathbf{1 . 7 0}$ & $\mathbf{9 0 . 0 8}$ & $\mathbf{2 . 9 7}$ & $\mathbf{1 2 . 1 9}$ & $\mathbf{3 . 8 8}$ \\
\hline
\end{tabular}

Table 5: Mean Dice similarity, Jaccard index, and Hausdorff distance and standard deviations (std) for the comparison of the segmentation results of Observer $1(\mathrm{O} 1)$ and Observer $2(\mathrm{O} 2)$ with MRK. The mean is denoted by $\mu$ and the for the standard deviation by $\sigma$.

\begin{tabular}{|c|c|c|c|c|c|c|c|c|c|c|c|c|}
\hline & \multicolumn{6}{|c|}{ MRK vs O1 } & \multicolumn{6}{|c|}{ MRK vs O2 } \\
\hline & \multicolumn{2}{|c|}{ Dice } & \multicolumn{2}{|c|}{ Jaccard } & \multicolumn{2}{|c|}{ Hausdorff } & \multicolumn{2}{|c|}{ Dice } & \multicolumn{2}{|c|}{ Jaccard } & \multicolumn{2}{|c|}{ Hausdorff } \\
\hline ID & $\mu(\%)$ & $\sigma(\%)$ & $\mu(\%)$ & $\sigma(\%)$ & $\mu(\%)$ & $\sigma(\%)$ & $\mu(\%)$ & $\sigma(\%)$ & $\mu(\%)$ & $\sigma(\%)$ & $\mu(\%)$ & $\sigma(\%)$ \\
\hline 1 & 92.70 & 1.19 & 86.41 & 2.06 & 10.43 & 2.43 & 92.40 & 1.80 & 85.92 & 3.09 & 9.35 & 2.63 \\
\hline 2 & 92.19 & 1.48 & 85.55 & 2.53 & 12.02 & 2.78 & 89.64 & 1.93 & 81.27 & 3.15 & 16.05 & 3.27 \\
\hline 3 & 92.44 & 2.82 & 86.07 & 4.54 & 12.64 & 4.39 & 90.92 & 3.57 & 83.53 & 5.72 & 13.65 & 4.51 \\
\hline 4 & 93.21 & 1.98 & 87.35 & 3.44 & 13.93 & 3.34 & 92.55 & 2.14 & 86.21 & 3.64 & 14.24 & 2.87 \\
\hline 5 & 93.08 & 3.38 & 87.23 & 5.59 & 13.49 & 5.68 & 92.17 & 2.90 & 85.61 & 4.91 & 14.11 & 4.92 \\
\hline 6 & 94.65 & 1.32 & 89.88 & 2.35 & 13.98 & 3.26 & 89.83 & 1.48 & 81.57 & 2.44 & 26.61 & 4.39 \\
\hline 7 & 92.81 & 3.18 & 86.75 & 5.34 & 11.42 & 4.64 & 92.19 & 3.35 & 85.68 & 5.57 & 11.30 & 3.95 \\
\hline 8 & 93.85 & 1.48 & 88.45 & 2.60 & 15.49 & 3.87 & 93.58 & 1.50 & 87.97 & 2.62 & 14.84 & 3.80 \\
\hline 9 & 93.57 & 1.20 & 87.94 & 2.12 & 13.69 & 3.56 & 93.78 & 1.19 & 88.31 & 2.09 & 12.96 & 3.59 \\
\hline 10 & 93.16 & 1.81 & 87.25 & 3.18 & 16.37 & 4.27 & 93.10 & 2.00 & 87.15 & 3.49 & 16.02 & 4.17 \\
\hline 11 & 92.30 & 1.50 & 85.73 & 2.58 & 12.99 & 3.11 & 92.93 & 1.10 & 86.81 & 1.92 & 11.72 & 2.85 \\
\hline 12 & 95.50 & 1.96 & 91.45 & 3.45 & 13.74 & 4.58 & 95.22 & 1.87 & 90.93 & 3.32 & 14.32 & 5.28 \\
\hline Average & 93.29 & 1.94 & 87.50 & 3.31 & 13.35 & $\mathbf{3 . 8 3}$ & 92.36 & 2.07 & 85.91 & 3.50 & 14.60 & 3.85 \\
\hline
\end{tabular}


Table 6: Mean Dice similarity, Jaccard index, and Hausdorff distance and standard deviations (std) for the comparison of the segmentation results of Observer 1 (O1) and Observer 2 (O2) UNL. The mean is denoted by $\mu$ and the for the standard deviation by $\sigma$.

\begin{tabular}{|r|r|r|r|r|r|r|r|r|r|r|r|r|}
\hline & \multicolumn{9}{|c|}{ UNL vs O1 } & \multicolumn{4}{c|}{ UNL vs O2 } \\
\hline & \multicolumn{2}{|c|}{ Dice } & \multicolumn{2}{|c|}{ Jaccard } & \multicolumn{2}{c|}{ Hausdorff } & \multicolumn{2}{|c|}{ Dice } & \multicolumn{3}{c|}{ Jaccard } & \multicolumn{2}{c|}{ Hausdorff } \\
\hline ID & $\mu(\%)$ & $\sigma(\%)$ & $\mu(\%)$ & $\sigma(\%)$ & $\mu(\%)$ & $\sigma(\%)$ & $\mu(\%)$ & $\sigma(\%)$ & $\mu(\%)$ & $\sigma(\%)$ & $\mu(\%)$ & $\sigma(\%)$ \\
\hline 1 & 90.24 & 1.80 & 82.26 & 3.02 & 13.94 & 2.86 & 89.78 & 1.42 & 81.49 & 2.34 & 15.53 & 2.65 \\
\hline 2 & 90.18 & 1.90 & 82.17 & 3.12 & 20.14 & 5.86 & 90.39 & 2.81 & 82.59 & 4.60 & 20.74 & 6.98 \\
\hline 3 & 93.26 & 2.75 & 87.50 & 4.73 & 12.11 & 4.56 & 88.31 & 3.40 & 79.23 & 5.48 & 16.94 & 4.18 \\
\hline 4 & 96.25 & 1.02 & 92.79 & 1.88 & 9.42 & 2.30 & 93.42 & 2.96 & 87.79 & 5.02 & 13.04 & 4.58 \\
\hline 5 & 94.79 & 4.30 & 90.36 & 6.73 & 11.14 & 6.01 & 93.00 & 3.89 & 87.15 & 6.40 & 12.23 & 5.70 \\
\hline 6 & 92.50 & 2.31 & 86.12 & 4.01 & 24.00 & 6.24 & 95.51 & 1.74 & 91.46 & 3.13 & 13.87 & 5.42 \\
\hline 7 & 95.59 & 1.17 & 91.57 & 2.15 & 8.34 & 2.13 & 95.65 & 1.19 & 91.69 & 2.17 & 8.03 & 2.09 \\
\hline 8 & 91.16 & 9.56 & 84.88 & 13.15 & 21.43 & 23.21 & 90.43 & 9.53 & 83.63 & 12.94 & 22.59 & 22.95 \\
\hline 9 & 91.22 & 2.42 & 83.94 & 4.07 & 20.98 & 7.59 & 91.25 & 2.57 & 84.01 & 4.33 & 20.77 & 8.38 \\
\hline 10 & 88.33 & 8.31 & 80.01 & 12.57 & 32.14 & 23.46 & 88.49 & 7.83 & 80.16 & 11.90 & 31.45 & 22.05 \\
\hline 11 & 93.52 & 1.10 & 87.84 & 1.93 & 9.73 & 1.29 & 94.07 & 0.57 & 88.82 & 1.02 & 8.68 & 1.18 \\
\hline 12 & 95.32 & 1.08 & 91.07 & 1.96 & 13.52 & 3.16 & 96.23 & 1.24 & 92.77 & 2.27 & 11.78 & 3.57 \\
\hline Average & $\mathbf{9 2 . 6 9}$ & $\mathbf{3 . 1 4}$ & $\mathbf{8 6 . 7 1}$ & $\mathbf{4 . 9 4}$ & $\mathbf{1 6 . 4 1}$ & $\mathbf{7 . 3 9}$ & $\mathbf{9 2 . 2 1}$ & $\mathbf{3 . 2 6}$ & $\mathbf{8 5 . 9 0}$ & $\mathbf{5 . 1 3}$ & $\mathbf{1 6 . 3 1}$ & $\mathbf{7 . 4 8}$ \\
\hline
\end{tabular}

Table 7: Mean Dice similarity, Jaccard index, and Hausdorff distance and standard deviations (std) for the comparison of the segmentation results of Observer $1(\mathrm{O} 1)$ and Observer $2(\mathrm{O} 2)$ with PAP. The mean is denoted by $\mu$ and the for the standard deviation by $\sigma$.

\begin{tabular}{|r|r|r|r|r|r|r|r|r|r|r|r|r|}
\hline & \multicolumn{9}{|c|}{ PAP vs O1 } & \multicolumn{5}{c|}{ PAP vs O2 } \\
\hline & \multicolumn{2}{|c|}{ Dice } & \multicolumn{2}{|c|}{ Jaccard } & \multicolumn{2}{c|}{ Hausdorff } & \multicolumn{2}{c|}{ Dice } & \multicolumn{3}{c|}{ Jaccard } & \multicolumn{2}{c|}{ Hausdorff } \\
\hline ID & $\mu(\%)$ & $\sigma(\%)$ & $\mu(\%)$ & $\sigma(\%)$ & $\mu(\%)$ & $\sigma(\%)$ & $\mu(\%)$ & $\sigma(\%)$ & $\mu(\%)$ & $\sigma(\%)$ & $\mu(\%)$ & $\sigma(\%)$ \\
\hline 1 & 70.87 & 3.52 & 54.99 & 4.15 & 46.70 & 5.43 & 70.99 & 3.49 & 55.13 & 4.08 & 48.19 & 6.48 \\
\hline 2 & 85.62 & 2.95 & 74.97 & 4.50 & 25.68 & 4.34 & 86.41 & 2.53 & 76.15 & 3.89 & 26.18 & 4.91 \\
\hline 3 & 89.55 & 2.78 & 81.18 & 4.60 & 16.17 & 3.61 & 88.21 & 2.52 & 79.00 & 3.99 & 18.77 & 4.71 \\
\hline 4 & 94.66 & 0.89 & 89.87 & 1.60 & 13.07 & 3.14 & 91.11 & 2.73 & 83.77 & 4.46 & 18.63 & 4.27 \\
\hline 5 & 92.69 & 10.11 & 87.40 & 11.08 & 14.10 & 21.13 & 92.45 & 9.73 & 86.95 & 11.32 & 13.98 & 20.48 \\
\hline 6 & 88.74 & 2.03 & 79.82 & 3.19 & 26.51 & 4.78 & 93.47 & 2.67 & 87.85 & 4.51 & 17.75 & 6.16 \\
\hline 7 & 85.45 & 9.45 & 75.45 & 10.73 & 25.42 & 31.31 & 84.16 & 9.25 & 73.44 & 10.31 & 26.72 & 31.41 \\
\hline 8 & 64.08 & 15.80 & 49.05 & 16.77 & 72.30 & 33.58 & 63.53 & 15.75 & 48.41 & 16.51 & 73.77 & 33.19 \\
\hline 9 & 86.59 & 13.87 & 78.35 & 16.88 & 43.90 & 64.46 & 87.82 & 14.40 & 80.48 & 17.54 & 39.22 & 65.81 \\
\hline 10 & 90.09 & 8.83 & 82.84 & 11.22 & 25.53 & 27.87 & 89.45 & 8.78 & 81.77 & 11.16 & 26.64 & 27.43 \\
\hline 11 & 90.96 & 7.34 & 84.01 & 9.16 & 20.38 & 30.71 & 91.36 & 7.54 & 84.73 & 9.50 & 19.65 & 31.02 \\
\hline 12 & 93.60 & 9.91 & 89.06 & 12.23 & 17.11 & 25.18 & 93.30 & 9.95 & 88.56 & 12.29 & 18.28 & 24.96 \\
\hline Average & $\mathbf{8 6 . 0 7}$ & $\mathbf{7 . 2 9}$ & $\mathbf{7 7 . 2 5}$ & $\mathbf{8 . 8 4}$ & $\mathbf{2 8 . 9 1}$ & $\mathbf{2 1 . 3 0}$ & $\mathbf{8 6 . 0 2}$ & $\mathbf{7 . 4 5}$ & $\mathbf{7 7 . 1 9}$ & $\mathbf{9 . 1 3}$ & $\mathbf{2 8 . 9 8}$ & $\mathbf{2 1 . 7 4}$ \\
\hline
\end{tabular}


Table 8: Mean Dice similarity, Jaccard index, and Hausdorff distance and standard deviations (std) for the comparison of the segmentation results of Observer $1(\mathrm{O} 1)$ and Observer 2 (O2) with KAT. The mean is denoted by $\mu$ and the for the standard deviation by $\sigma$.

\begin{tabular}{|r|c|c|c|c|c|c|c|c|c|c|c|c|}
\hline & \multicolumn{9}{|c|}{ KAT vs O1 } & \multicolumn{5}{c|}{ PAP vs O2 } \\
\hline & \multicolumn{2}{|c|}{ Dice } & \multicolumn{2}{|c|}{ Jaccard } & \multicolumn{2}{c|}{ Hausdorff } & \multicolumn{2}{|c|}{ Dice } & \multicolumn{3}{c|}{ Jaccard } & \multicolumn{2}{c|}{ Hausdorff } \\
\hline ID & $\mu(\%)$ & $\sigma(\%)$ & $\mu(\%)$ & $\sigma(\%)$ & $\mu(\%)$ & $\sigma(\%)$ & $\mu(\%)$ & $\sigma(\%)$ & $\mu(\%)$ & $\sigma(\%)$ & $\mu(\%)$ & $\sigma(\%)$ \\
\hline 1 & 82.73 & 4.19 & 70.75 & 5.82 & 25.36 & 4.11 & 82.87 & 4.64 & 71.00 & 6.50 & 22.32 & 3.39 \\
\hline 2 & N.A. & N.A. & N.A. & N.A. & N.A. & N.A. & N.A. & N.A. & N.A. & N.A. & N.A. & N.A. \\
\hline 3 & N.A. & N.A. & N.A. & N.A. & N.A. & N.A. & N.A. & N.A. & N.A. & N.A. & N.A. & N.A. \\
\hline 4 & N.A. & N.A. & N.A. & N.A. & N.A. & N.A. & N.A. & N.A. & N.A. & N.A. & N.A. & N.A. \\
\hline 5 & N.A. & N.A. & N.A. & N.A. & N.A. & N.A. & N.A. & N.A. & N.A. & N.A. & N.A. & N.A. \\
\hline 6 & N.A. & N.A. & N.A. & N.A. & N.A. & N.A. & N.A. & N.A. & N.A. & N.A. & N.A. & N.A. \\
\hline 7 & 90.21 & 3.38 & 82.33 & 5.53 & 14.75 & 4.23 & 89.49 & 3.50 & 81.15 & 5.69 & 15.43 & 4.69 \\
\hline 8 & 89.32 & 4.07 & 80.93 & 6.54 & 23.83 & 9.42 & 88.99 & 4.01 & 80.39 & 6.49 & 25.35 & 9.10 \\
\hline 9 & 89.62 & 1.67 & 81.23 & 2.74 & 25.59 & 3.59 & 90.73 & 1.94 & 83.08 & 3.26 & 23.53 & 4.69 \\
\hline 10 & 88.69 & 3.94 & 79.88 & 6.15 & 22.67 & 9.24 & 88.68 & 3.80 & 79.87 & 5.96 & 24.11 & 9.12 \\
\hline 11 & 93.32 & 2.17 & 87.54 & 3.77 & 11.77 & 2.50 & 93.93 & 1.78 & 88.60 & 3.13 & 11.22 & 2.04 \\
\hline 12 & 89.49 & 2.50 & 81.06 & 4.08 & 23.47 & 5.81 & 88.68 & 2.81 & 79.77 & 4.55 & 24.62 & 6.06 \\
\hline Average & $\mathbf{8 9 . 0 5}$ & $\mathbf{3 . 1 3}$ & $\mathbf{8 0 . 5 3}$ & $\mathbf{4 . 9 5}$ & $\mathbf{2 1 . 0 6}$ & $\mathbf{5 . 5 6}$ & $\mathbf{8 9 . 0 5}$ & $\mathbf{3 . 2 1}$ & $\mathbf{8 0 . 5 5}$ & $\mathbf{5 . 0 8}$ & $\mathbf{2 0 . 9 4}$ & $\mathbf{5 . 5 8}$ \\
\hline
\end{tabular}




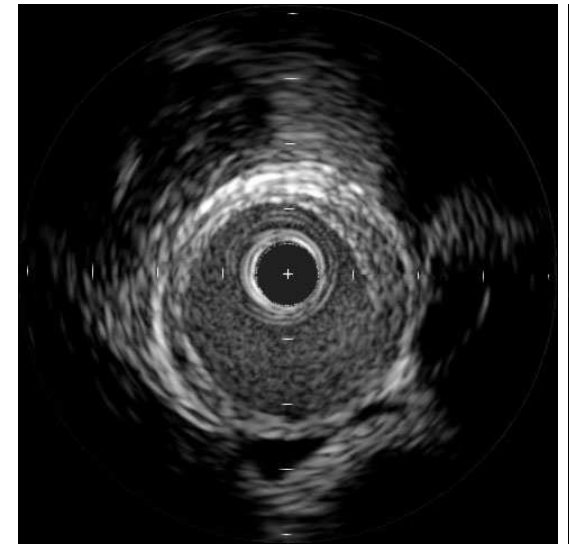

(a)

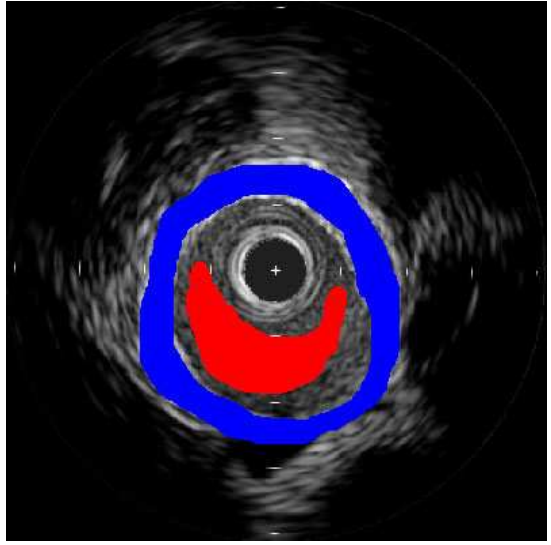

(b)

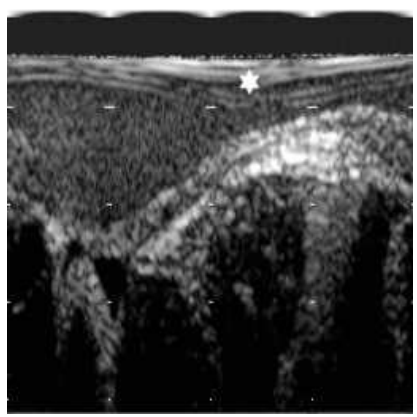

(c)

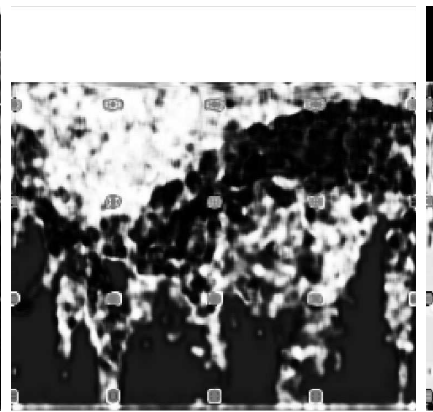

(d)

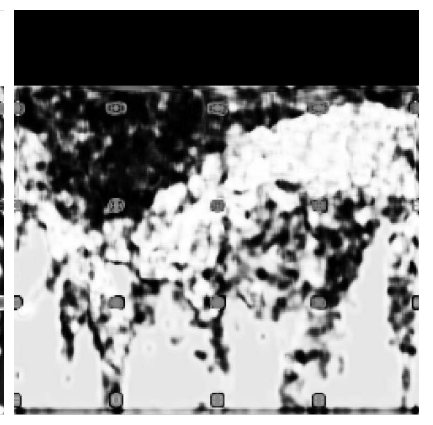

(e)

Figure 6: Examples depicting (a) the first frame of a sequence, (b) the corresponding user annotation for blood and non-blood (red and blue, respectively), (c) the corresponding polar B-mode representation, and the likelihood estimates for (d) lumen and (e) non-lumen. The star in (c) indicates the radius coordinate $\chi$ of the outer-most trace of the ringdown artifact selected by the user. 


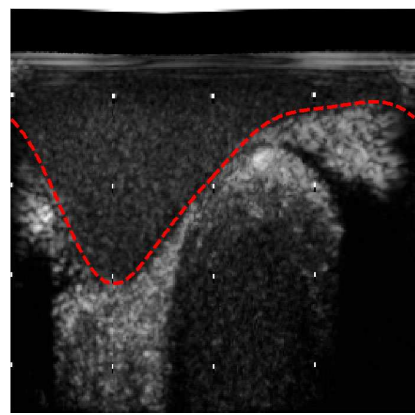

(a)

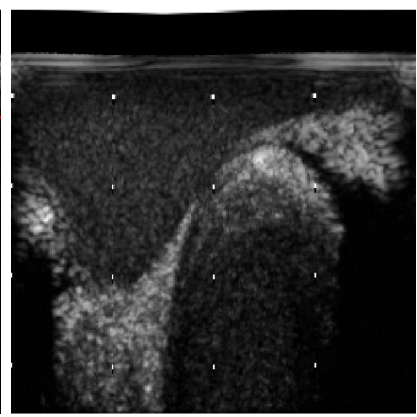

(b)

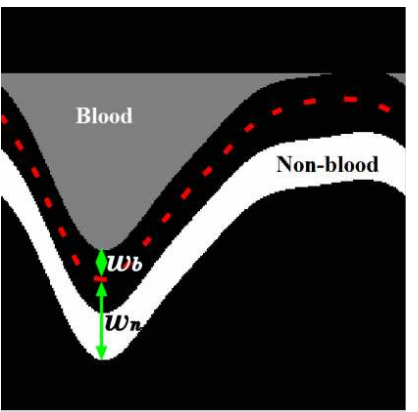

(c)

Figure 7: Examples depicting (a) the lumen segmentation result of a frame, (b) the next consecutive frame, and (c) the class mask which defines the regions considered as blood and non-blood.

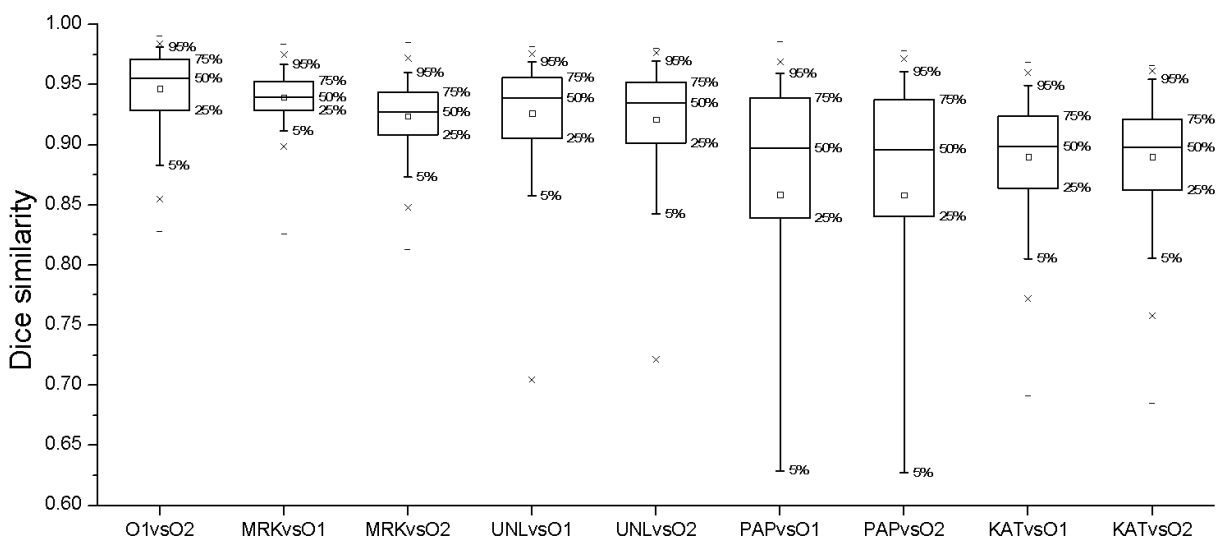

Figure 8: Box plot of the Dice similarity coefficient for the comparison of the segmentation results in all the sequences of the proposed method (MRK), Unal's method (UNL), Papadogiorgaki's method (PAP), and Katouzian's method (KAT) with the manual segmentation of two observers. 


\subsection{Results on a sequence with changes in appearance}

To evaluate the robustness of the MRK method respect to changes in the Bmode reconstruction parameters and large lumen shape changes, we performed an experiment using a modified version of Sequence 7 where we applied first a $90^{\circ}$ rotation and then changes in the dynamic range compression every 10 frames. Figure 20 depicts the segmentation result on the first frame of each modified subsequence. In this case the average frame segmentation time was $4.51 \mathrm{~s}$, the model was computed four times, and the total model computation time was $63.88 \mathrm{~s}$. The mean Dice similarity of the comparison with the manual segmentation was $92.84 \%$ with a standard deviation of $2.19 \%$. This results indicates the robustness of our method with respect to possible changes in appearance within an IVUS sequence due to changes in the B-mode reconstruction parameters, catheter rotations, or the natural changes on the physiology of the vessel.

\section{Discussion}

While the results obtained with the UNL method are comparable with the results our method, note that the MRK method presents a consistently smaller standard deviation which means that our method is more stable. In addition, the significance test determined that the results obtained with the MRK method are significant when compared with the other methods. In contrast to other methods, the MRK method does not require any supervised parameter tuning when changing between sequences with different IVUS frequencies or B-mode reconstruction parameters. Most of the frame segmentation time is used by the computation of the Law's texture energies. However, considering that this code was implemented in a MATLAB ${ }^{\circledR}$, we believe that the segmentation process time can be dramatically reduced by implementing the method using a lower level language such as $\mathrm{C}++$.

\subsection{Sensitivity analysis}

\subsubsection{Lumen contour parameters}

As mentioned in Sec. 3.2, the sharpness of the transition between classes is controlled by the parameter $\lambda$. The value of this parameter may have an impact in the accuracy of the segmentation curve. On the other hand, the number of Fourier coefficients $N_{k}$ determines the smoothness of the curve. To evaluate the sensitivity of our method with respect to these parameters, we performed segmentation of all the frames from the available IVUS sequences using different values for $\lambda$ and $N_{k}$, and computed the similarity of the results with the manual segmentation of 


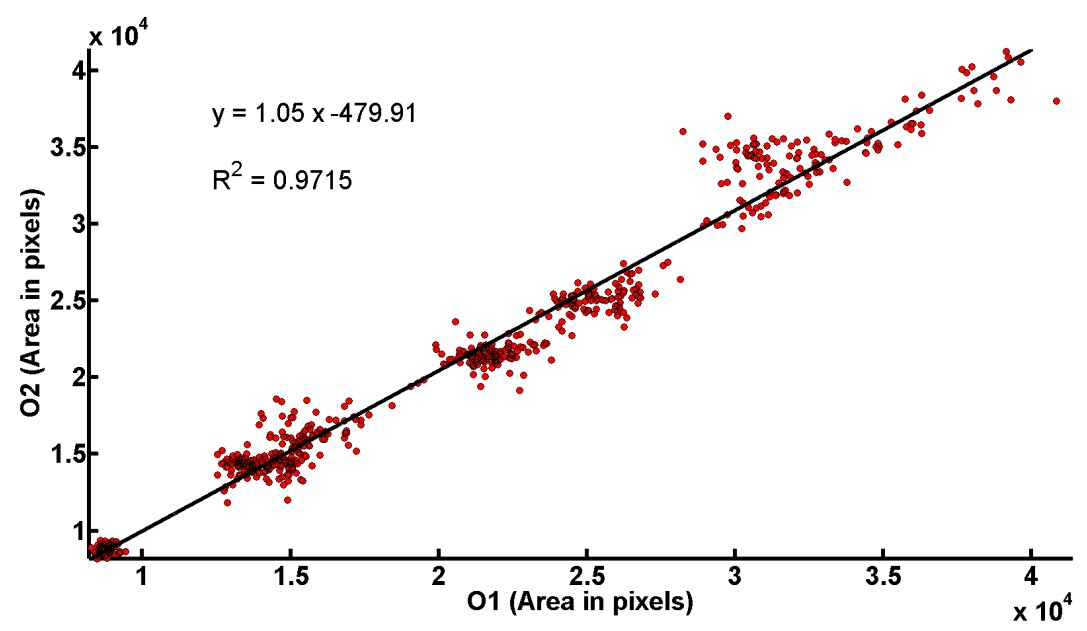

(a)

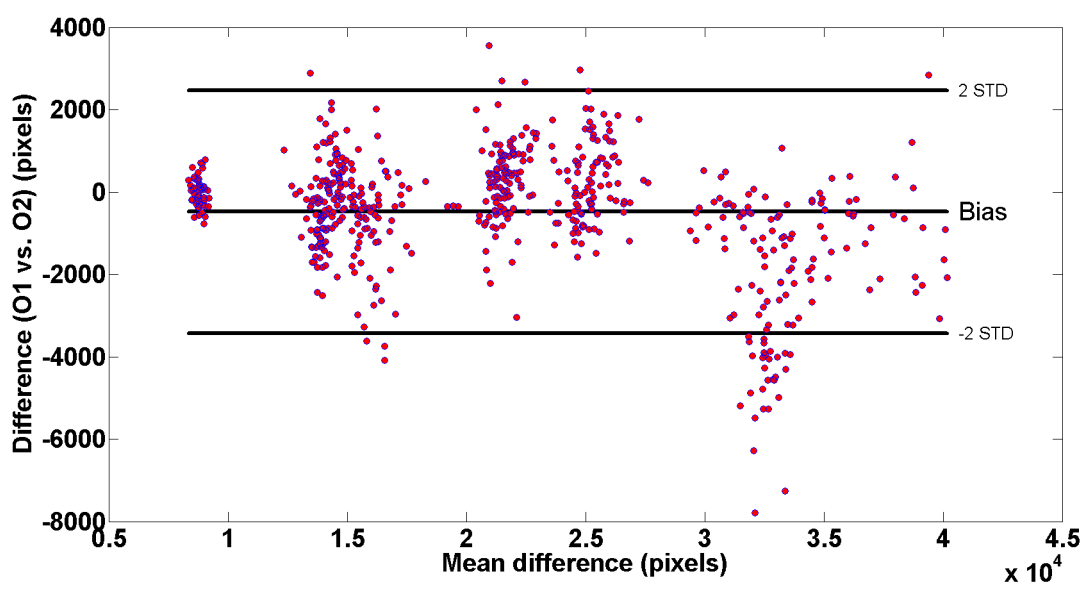

(b)

Figure 9: (a) Linear regression and (b) Bland-Altman plot for the comparison of the lumen areas segmented by Observer $1(\mathrm{O} 1)$ with the areas segmented by Observer $2(\mathrm{O} 2)$. 


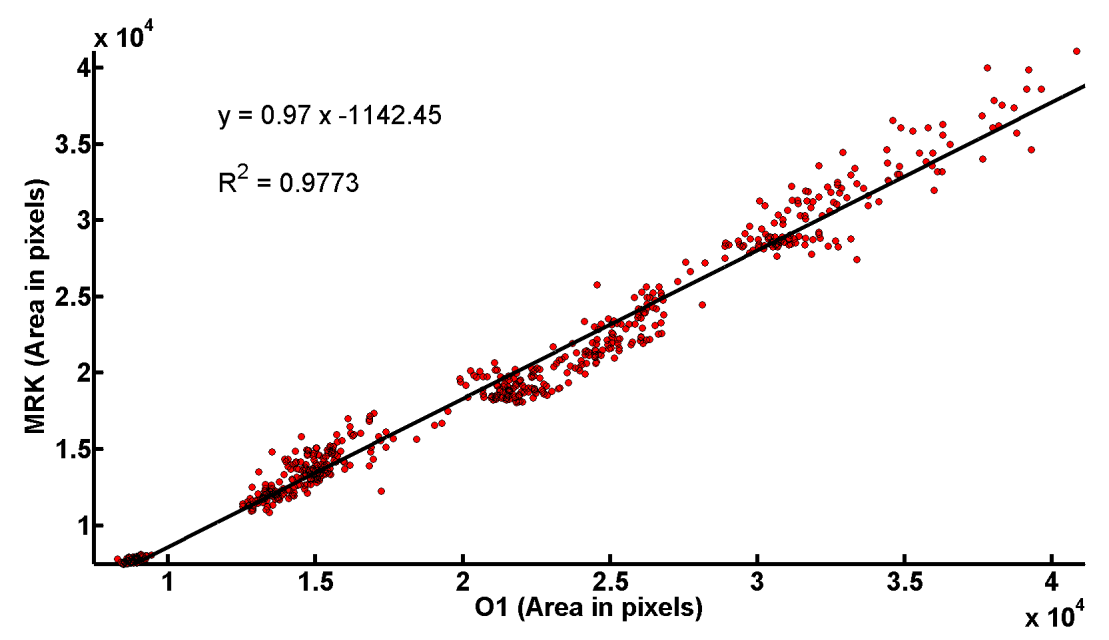

(a)

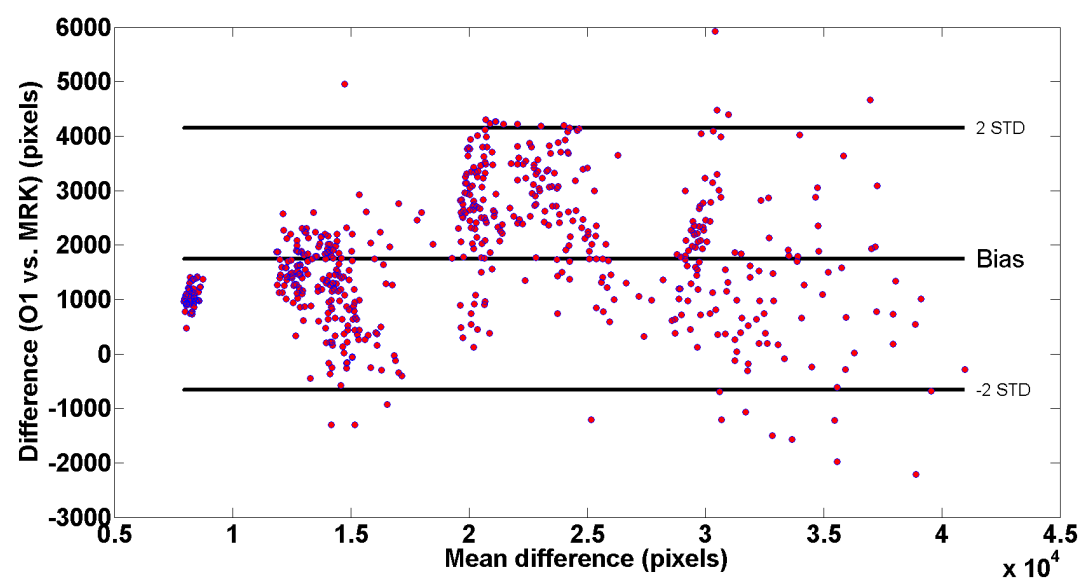

(b)

Figure 10: $(\mathrm{a}, \mathrm{b})$ Linear regression and (c,d) Bland-Altman plot for the comparison of the lumen areas segmented by Observer $1(\mathrm{O} 1)$ with the areas segmented with the MRK method. 


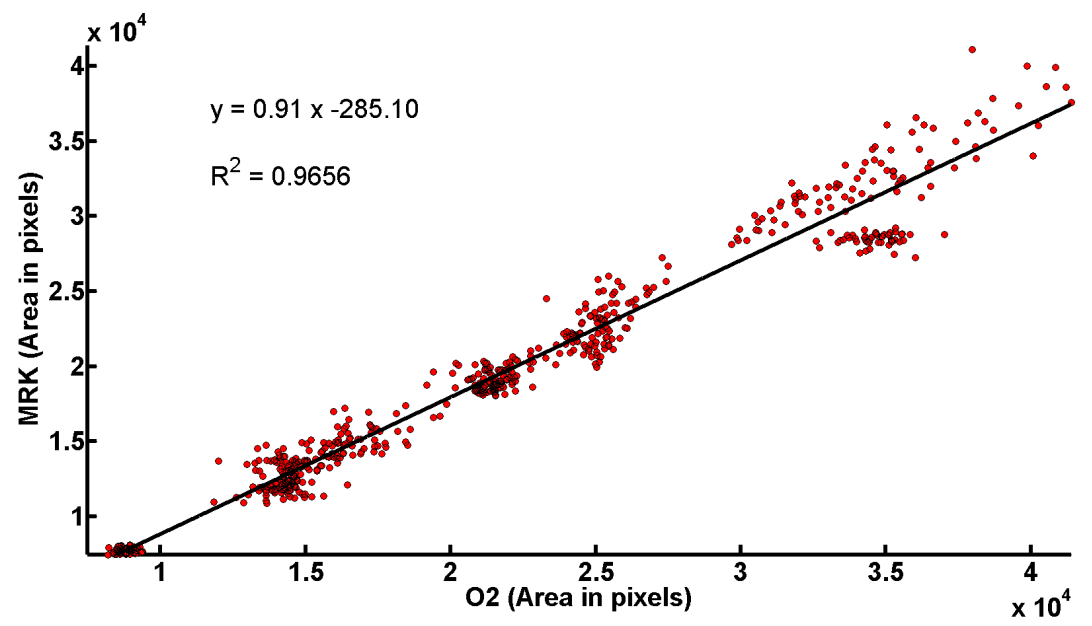

(a)

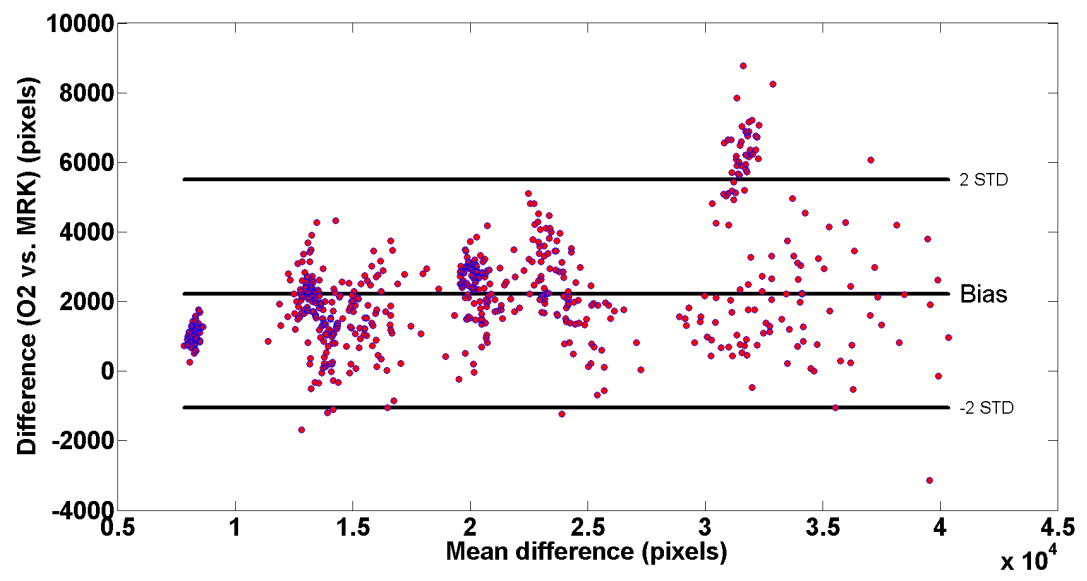

(b)

Figure 11: (a, b) Linear regression and (c,d) Bland-Altman plot for the comparison of the lumen areas segmented by Observer $2(\mathrm{O} 2)$ with the areas segmented with the MRK method. 


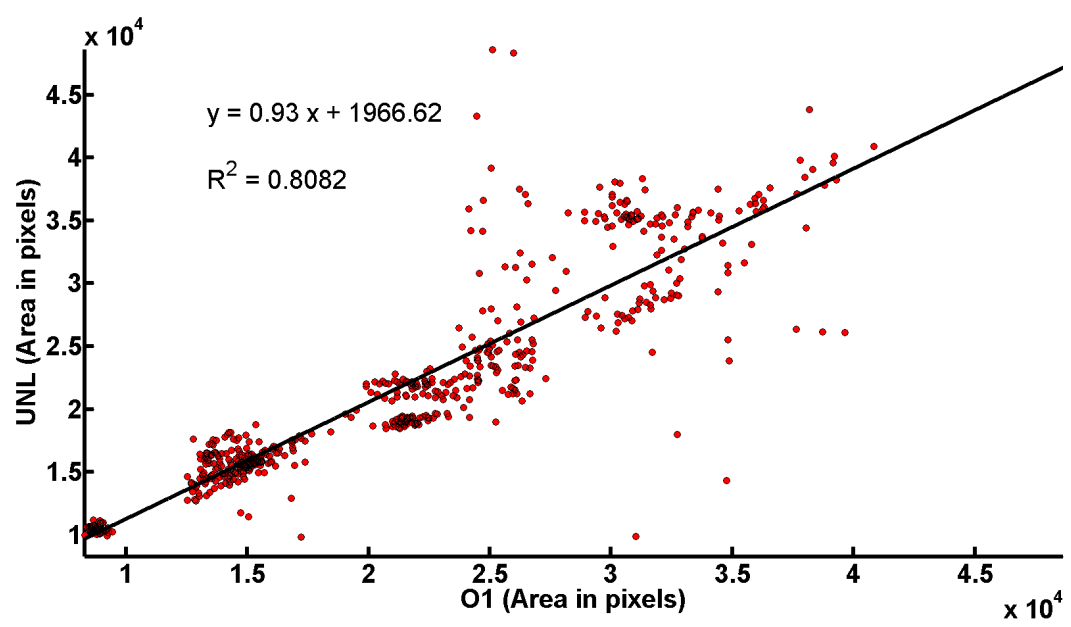

(a)

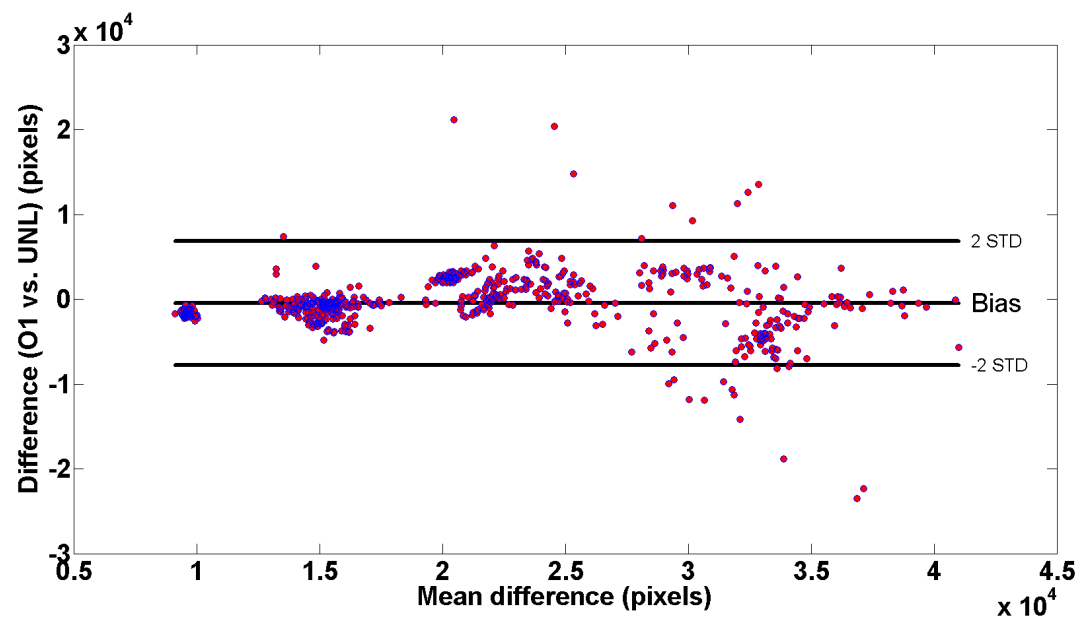

(b)

Figure 12: (a, b) Linear regression and (c,d) Bland-Altman plot for the comparison of the lumen areas segmented by Observer 1 (O1) with the areas segmented with the UNL method. 


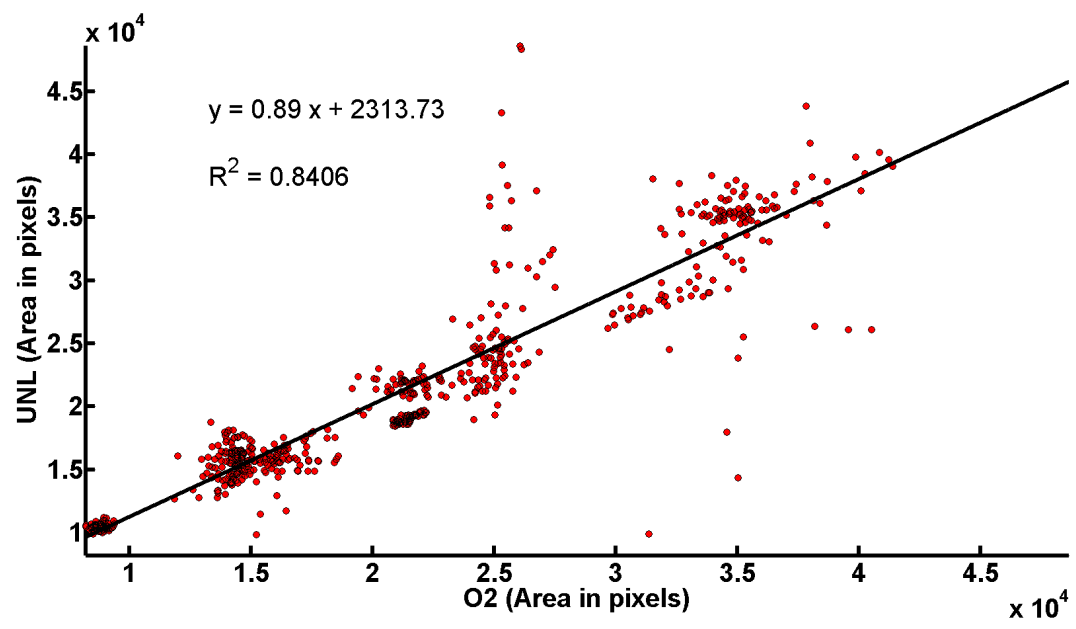

(a)

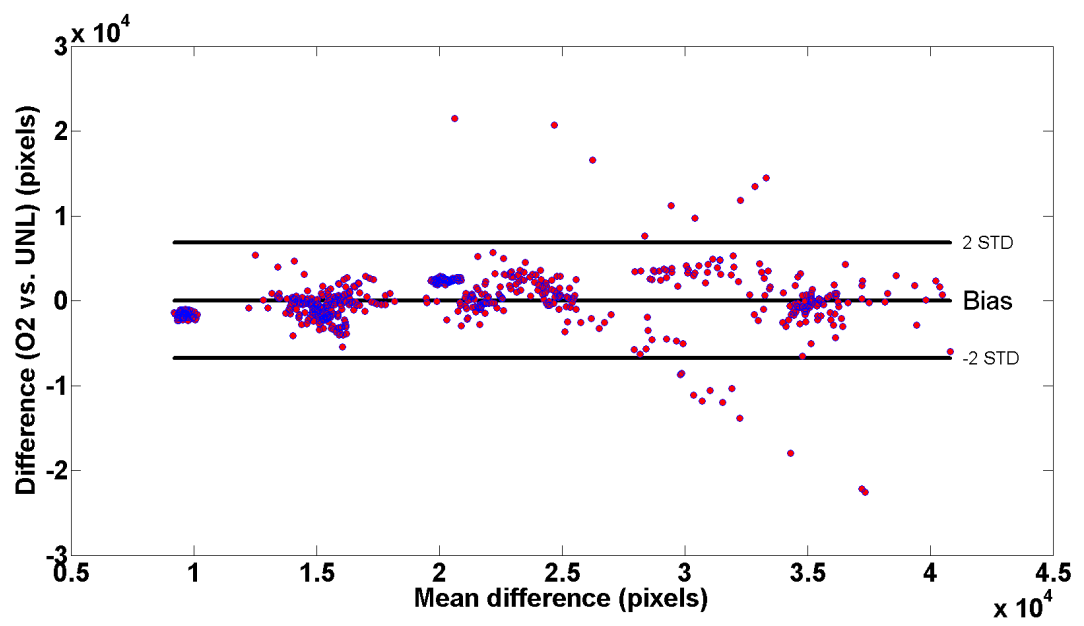

(b)

Figure 13: (a, b) Linear regression and (c,d) Bland-Altman plot for the comparison of the lumen areas segmented by Observer $2(\mathrm{O} 2)$ with the areas segmented with the UNL method. 


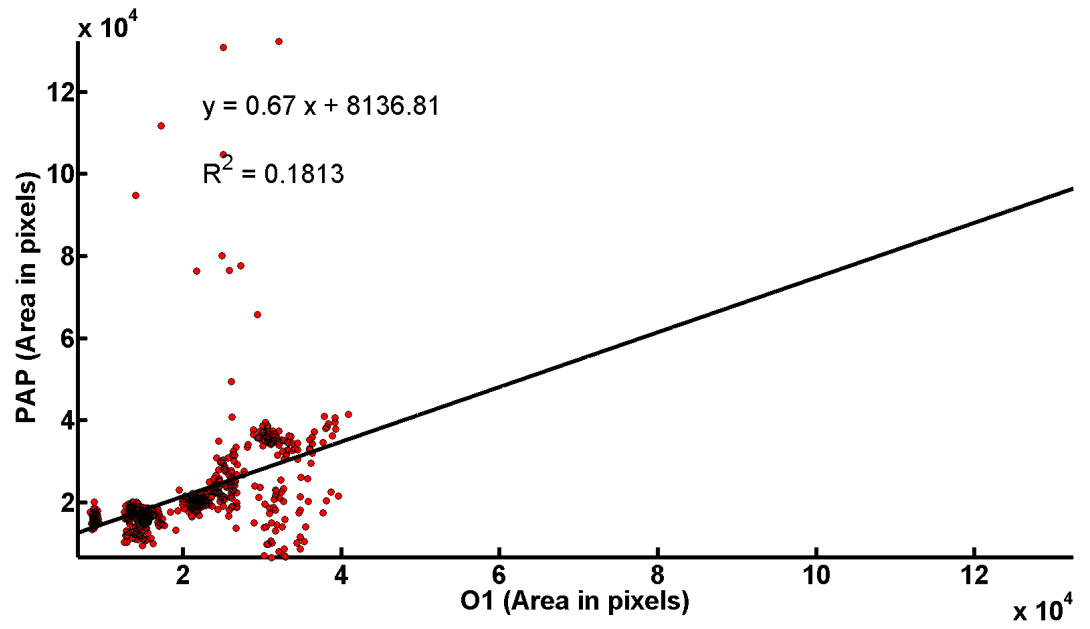

(a)

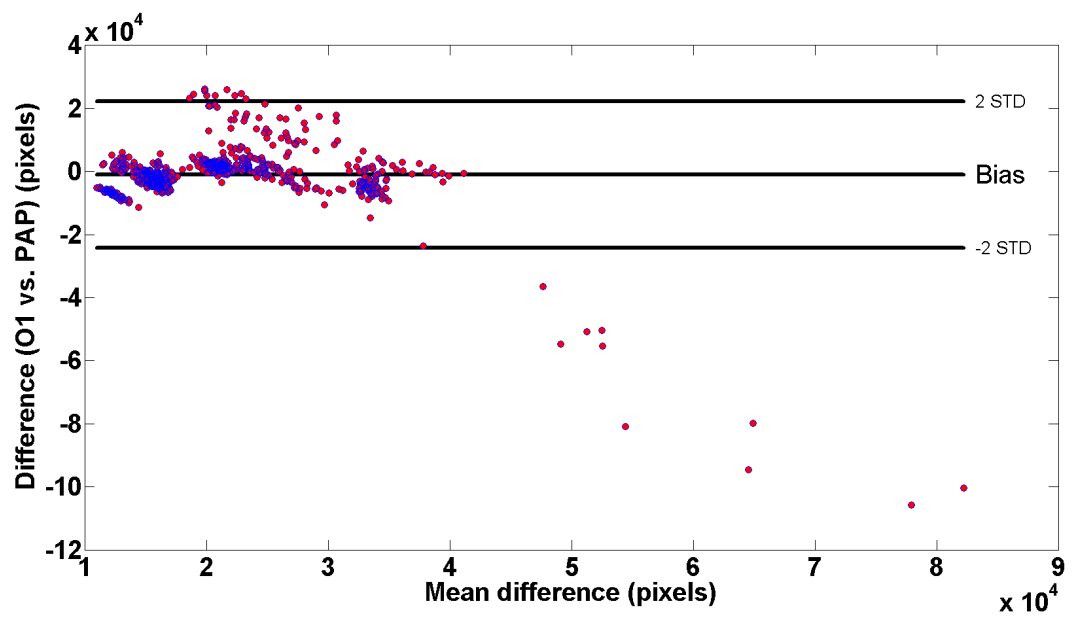

(b)

Figure 14: (a, b) Linear regression and (c,d) Bland-Altman plot for the comparison of the lumen areas segmented by Observer $1(\mathrm{O} 1)$ with the areas segmented with the PAP method. 


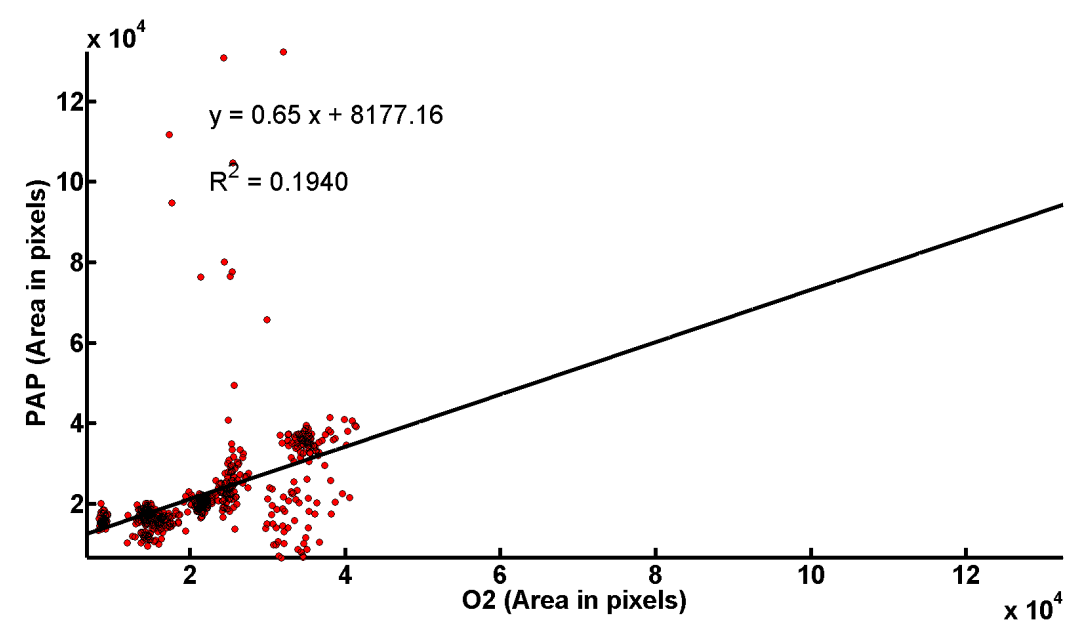

(a)

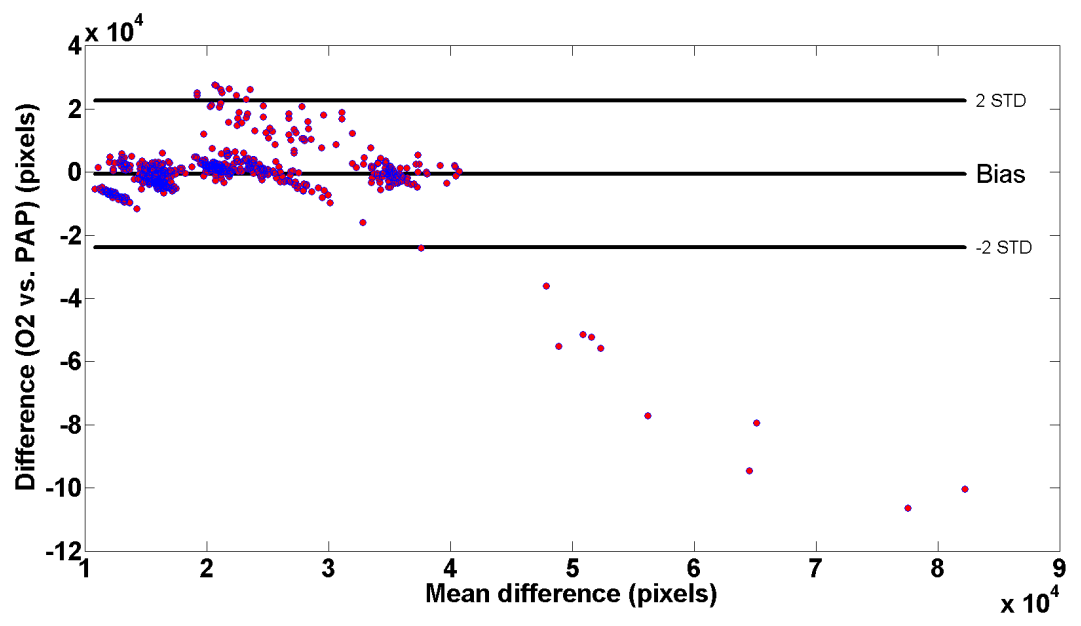

(b)

Figure 15: (a, b) Linear regression and (c,d) Bland-Altman plot for the comparison of the lumen areas segmented by Observer $2(\mathrm{O} 2)$ with the areas segmented with the PAP method. 


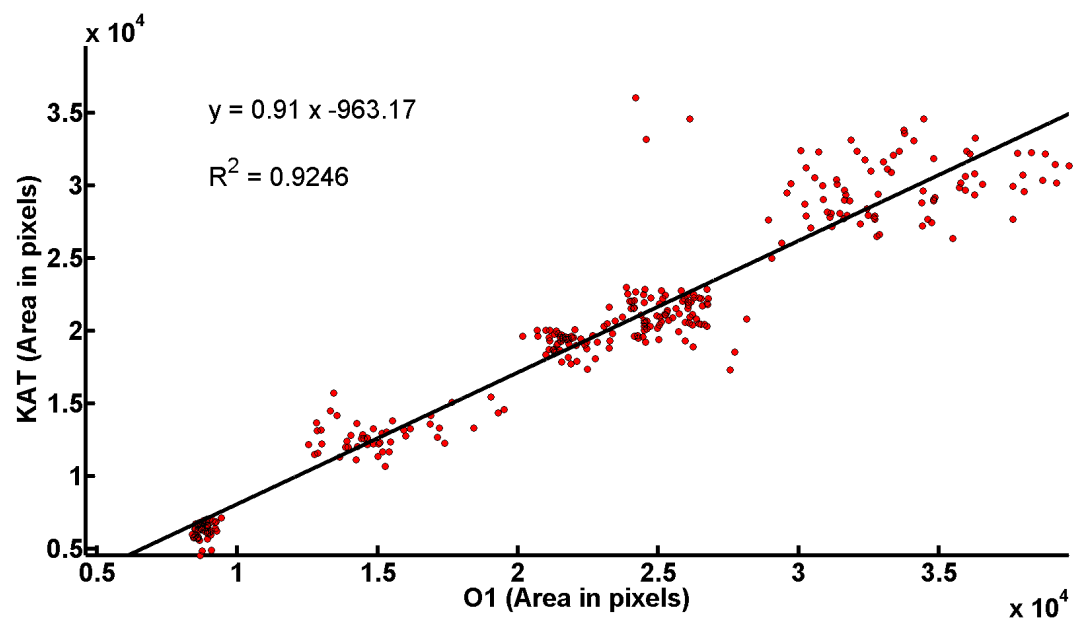

(a)

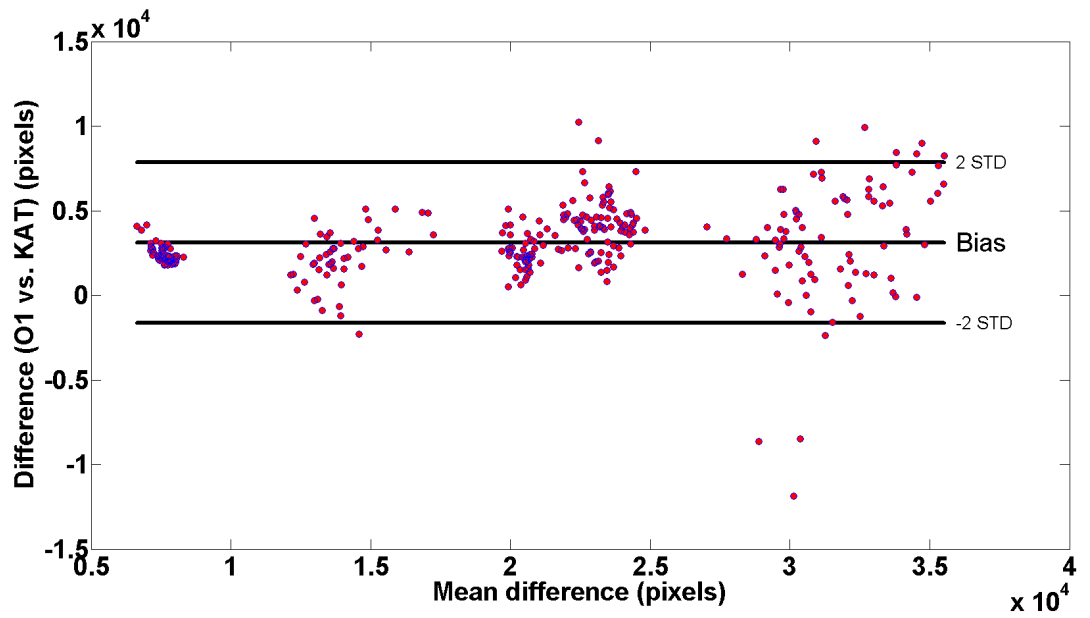

(b)

Figure 16: (a, b) Linear regression and (c,d) Bland-Altman plot for the comparison of the lumen areas segmented by Observer $1(\mathrm{O} 1)$ with the areas segmented with the KAT method. 


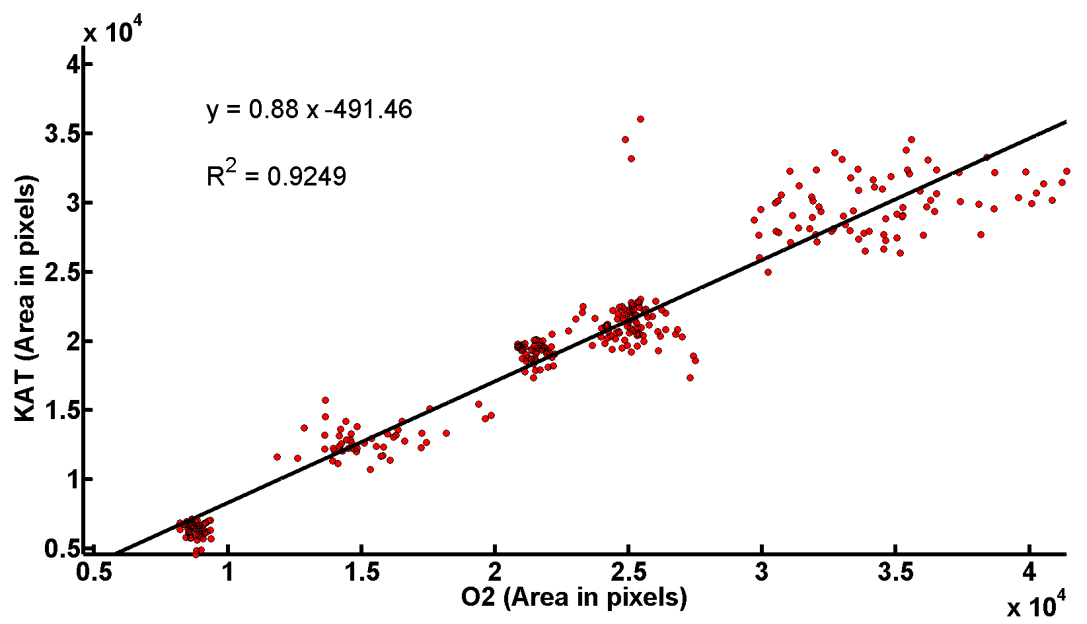

(a)

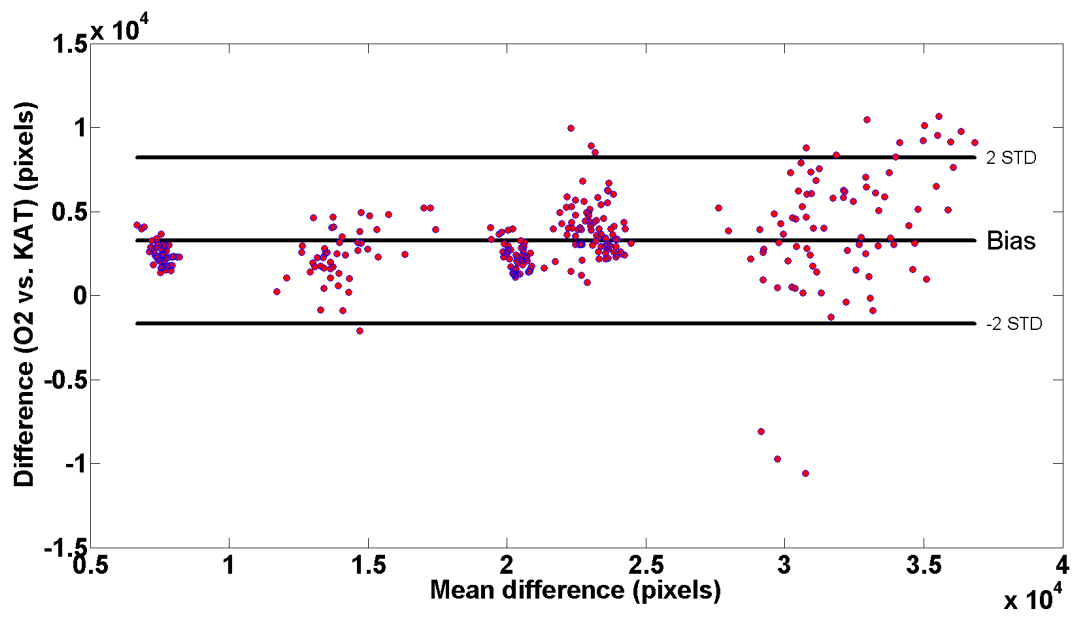

(b)

Figure 17: (a, b) Linear regression and (c,d) Bland-Altman plot for the comparison of the lumen areas segmented by Observer $2(\mathrm{O} 2)$ with the areas segmented with the KAT method. 

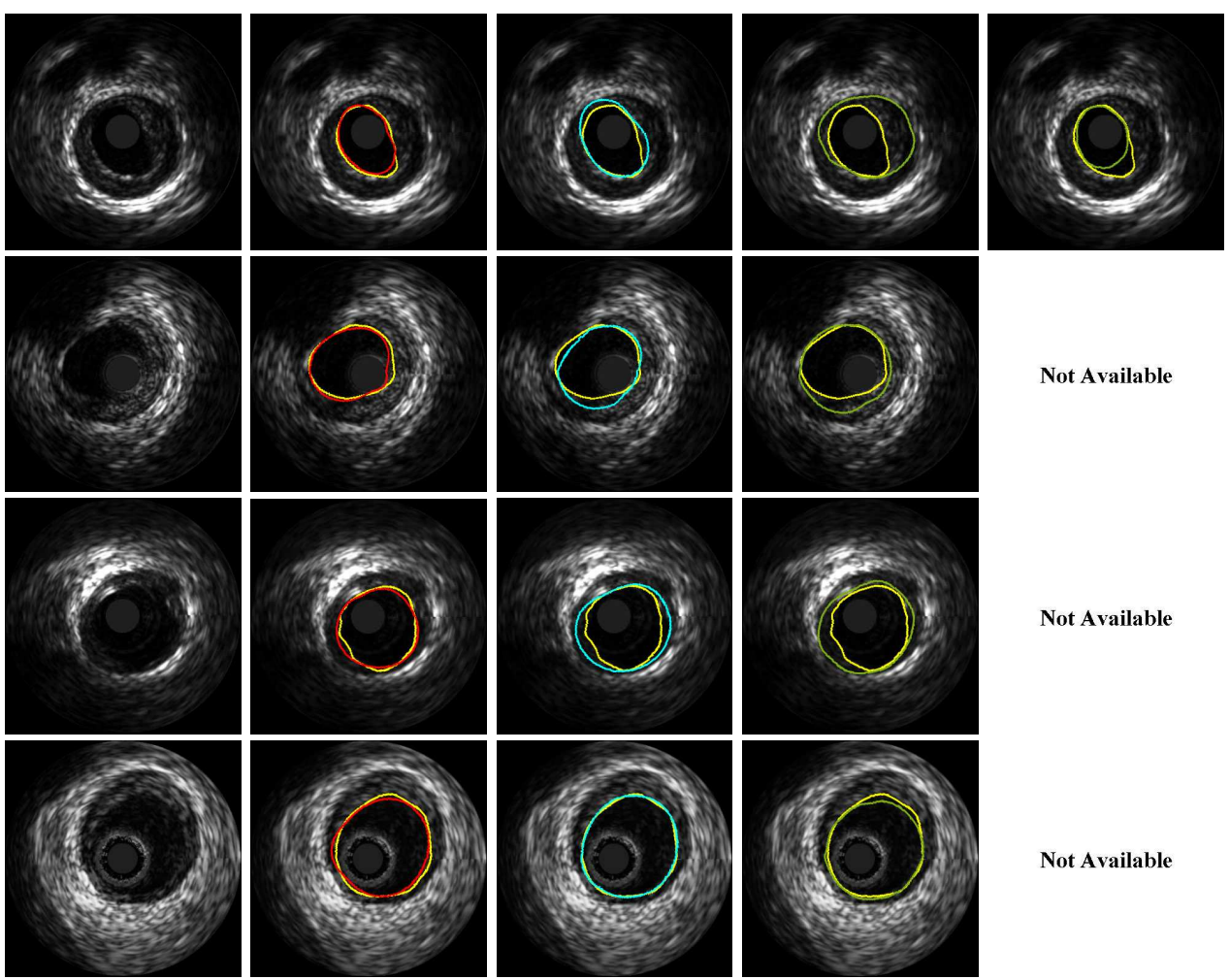

Not Available
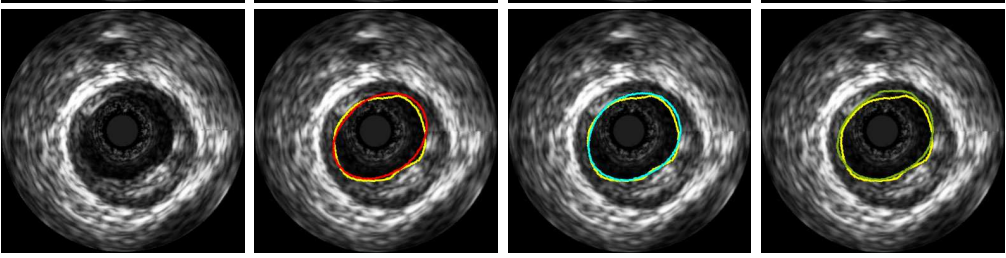

Not Available
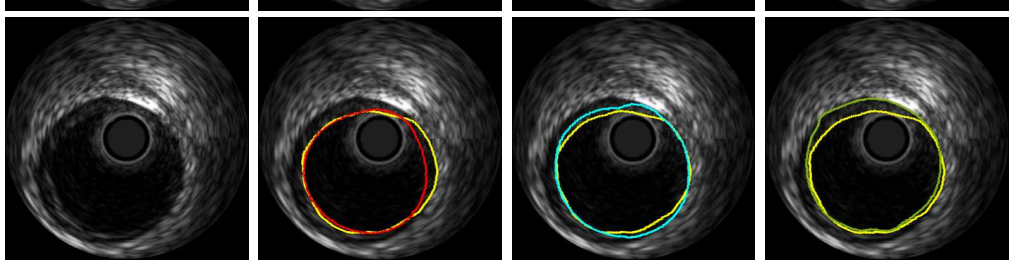

Not Available

Not Available

Figure 18: Examples of automatic segmentation results for $20 \mathrm{MHz}$ sequences along with the segmentation of Observer 1 (yellow line). The images correspond to the original frame and the segmentation results with the MRK, UNL, PAP, and KAT methods, from left to right, respectively. 

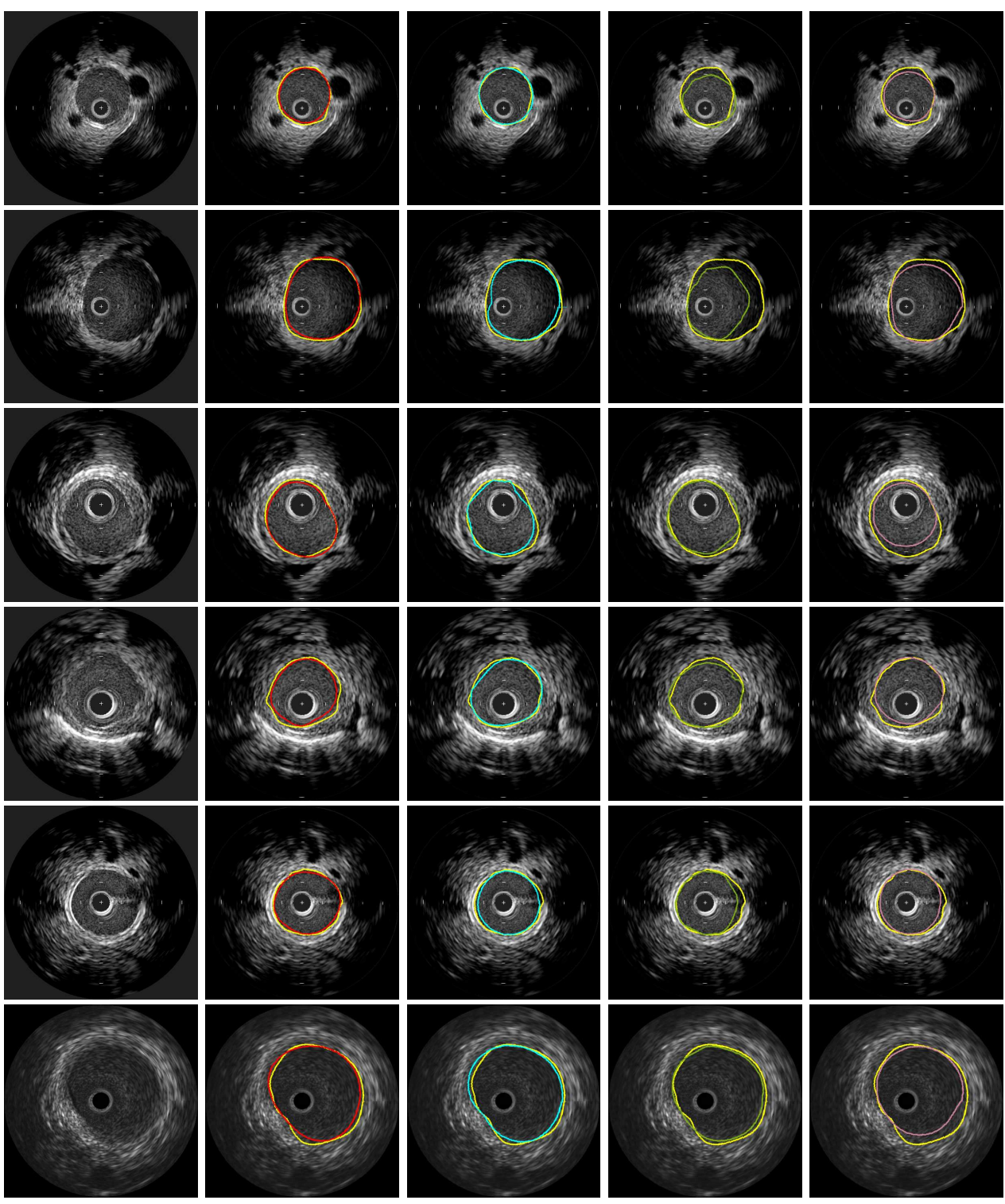

Figure 19: Examples of automatic segmentation results for $40 \mathrm{MHz}$ sequences along with the segmentation by Observer 1 (yellow line). The images correspond to the original frame and the segmentation results with the MRK, UNL, PAP, and KAT methods, from left to right, respectively. 


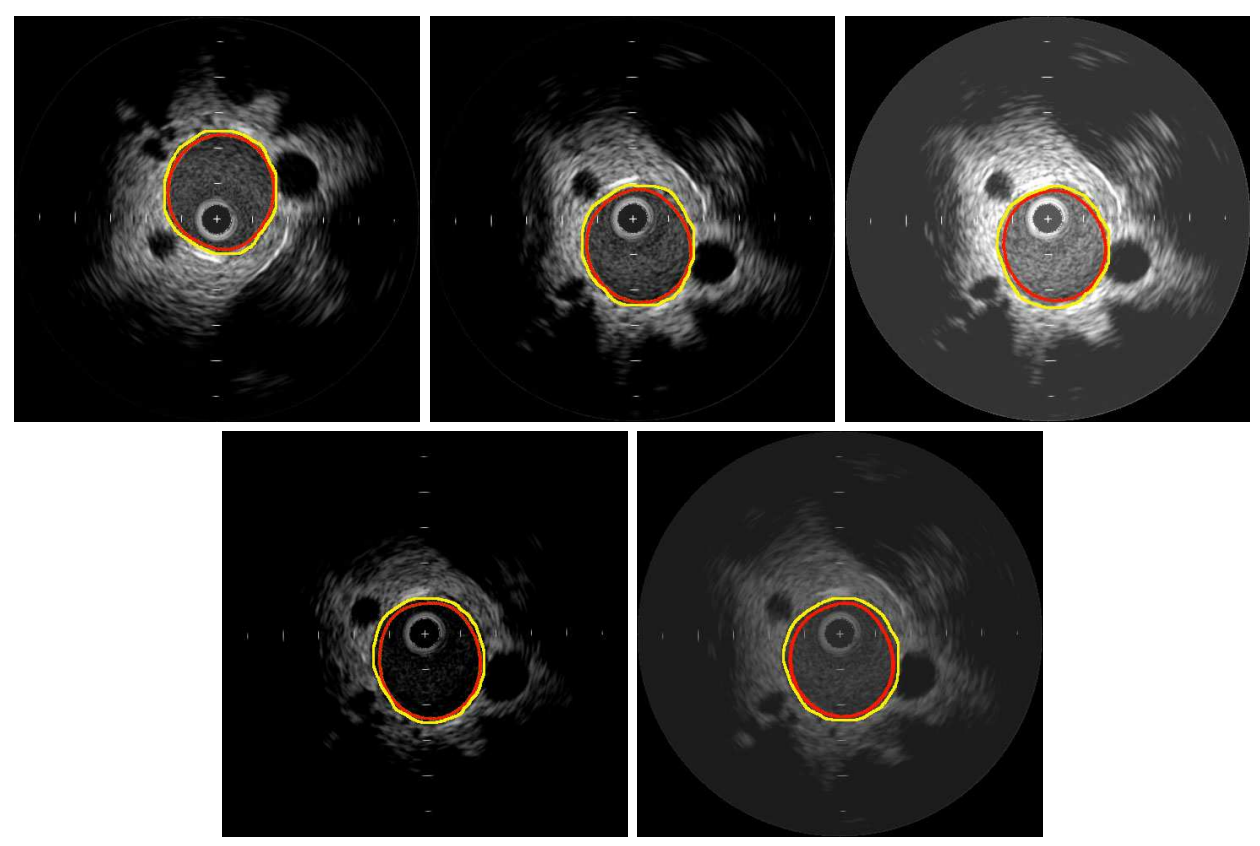

Figure 20: Segmentation results on the first frame of each modified subsequence.

an observer using the Dice coefficient (Fig. 21(a)). Note that as the value of $N_{k}$ increases, the segmentation results improve. However, since the number of variables of the cost function of Eq. (5) is related with the value of $N_{k}$, the time required to minimize the cost function that provides the segmentation depends on the value of $N_{k}$. Figure 21(b) depicts the average segmentation times for all the sequences using different values of $N_{k}$. Note that as the value of $N_{k}$ increases, the average time required to segment a frame also increases. Note that the value of the parameter $\lambda$ does not significatively affect the segmentation results as the value of $N_{k}$ increases.

\subsubsection{Consecutive frames similarity}

The proposed gray-level distribution change detection of the proposed MRK method is based on the assumption that the shape of the lumen of two consecutive frames is very similar. In order to quantitatively verify this observation and to determine the values of $w_{b}$ and $w_{n}$ we computed the Dice similarity coefficient Dice (1945) between the region defined as lumen by an observer for each pair of consecutive frames corresponding to the validation sequences described in Table 2. Figure 22(a) depicts the histogram of the obtained Dice similarities coefficients 
for the comparison of all the consecutive frames. Note that the similarity between consecutive frames is most of the time above $97 \%$ which supports our assumption. Similarly, we computed the maximum difference between the radial coordinate $r$ in of the pixels corresponding to the same angle $\theta$ belonging to the lumen contour on each pair of consecutive frames $\max \left(S_{i}\left(r, \theta_{j}, \vec{C}_{i}\right)-S_{i+1}\left(r, \theta_{j}, \vec{C}_{i+1}\right)\right) \forall j$. Figure 22(b) depicts the empirical distribution function of the maximum differences for all the frames of the validation set described in Table 2. Note that, for more than $90 \%$ of the frames the largest radial difference is less than 20 pixels.

\subsection{Results on IVUS images with artifacts}

Shadows may appear on the IVUS images as dark regions representing of low acoustic impedance. In general these artifacts are generated by calcified plaque or a region of tissue with low acoustic impedance proximal to the vessel. Shadows represent a challenge for $20 \mathrm{MHz}$ sequences because the low echogenicity of blood at this frequency generates gray level distributions on similar to those regions corresponding to shadows. However, since in our approach the lumen region is constrained by a curve it is not affected by these artifacts as long as there is a region with texture different from the lumen. Figures 23(a), 23(b) and 23(c) depicts a $20 \mathrm{MHz}$ cases presenting shadows proximal to the vessel lumen. Note that, the MRK method was able to provide an accurate segmentation because the existence of regions between the lumen and the shadow which constrain the evolution of the curve. Similarly, Fig. 23(d) depicts a $40 \mathrm{MHz}$ case which presents a large shadow proximal to the vessel lumen. Note that for this case, the MRK method was able to provide an accurate segmentation because of the evident difference between the texture of the lumen and the shadow region. In general, shadow artifacts do not represent a challenge for $40 \mathrm{MHz}$ sequences since the gray level intensities of the speckle present in blood are different from the homogeneously dark shadow regions.

Side branches are identified as the opening formed when the vessel being imaged bifurcates. This is visualized as an area with similar texture extending from the lumen in the near field towards the far field. Side branches represents a challenge for any segmentation method and in some cases even for observers because of the lack of an apparent change in the regions that indicates a change of interface. For these cases, the MRK method is not capable of providing a segmentation result similar with the segmentation of an observer. Figure 24 depicts an example of one case presenting a side branch with origin in the lumen. In this example, the curve that defines the lumen/wall interface of the MRK method move through 
this region because it is designed to keep inside the contour as much region with texture of blood as possible.

Similarly, for cases presenting regions of the vessel wall adjacent to the lumen contour with texture similar to the texture depicted in the lumen, the lack of an apparent change in the regions represents a challenge for automatic any segmentation method and observers. The MRK method may produce inaccurate segmentation results in this cases. Figure 25 depicts an example of two $40 \mathrm{MHz}$ frames in which the texture of the lumen is similar to the texture of a region of the wall. In these cases the MRK method performed incorrectly.

Guidewire artifacts are produced by the reverberation of the guidewire when interacting with the ultrasound beam. Depending on the location of the guidewire with respect to the ultrasound transducer, these artifacts appears in the IVUS images as bright thick spots or as series of partial rings followed by more distant shadowing behind the wire posing a challenge for the segmentation methods. Figure 26 depicts the segmentation result using MRK on images that present guidewire artifacts. Note that the MRK method is capable to provide accurate segmentation results (Figs. 26(a), 26(b), and 26(c)). However, for some cases where there is a region of the lumen occupied by the artifact the MRK method may perform incorrectly (Fig. 26(d)). 


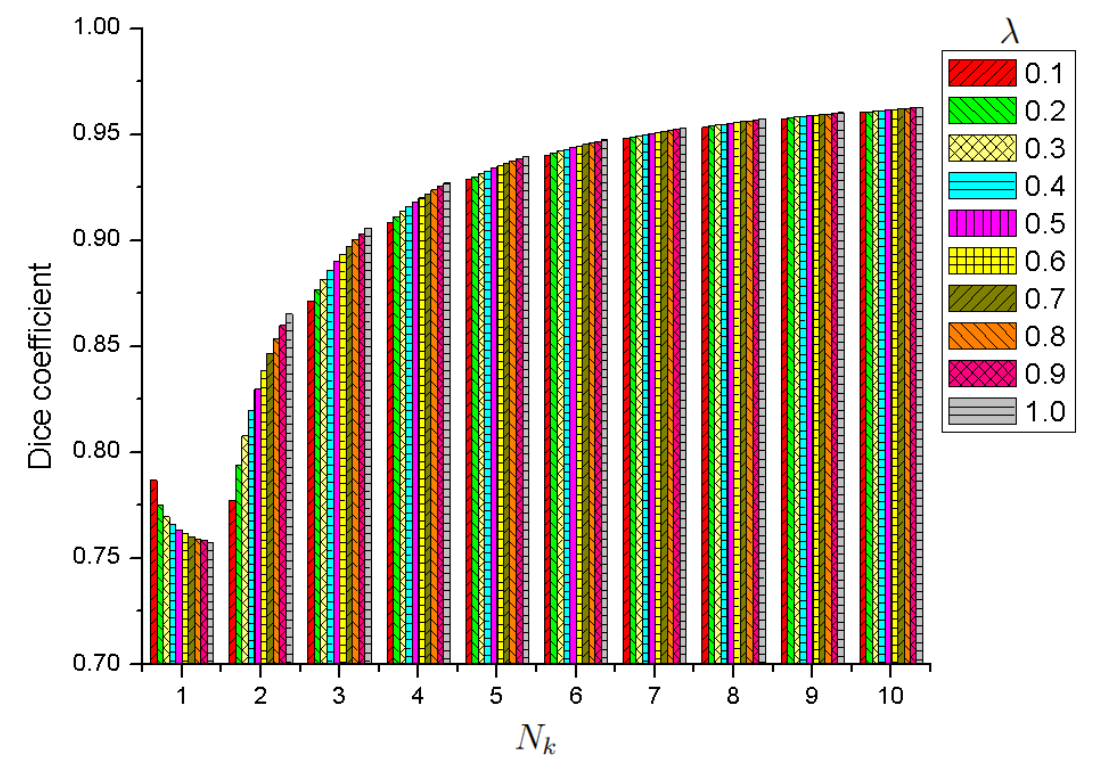

(a)

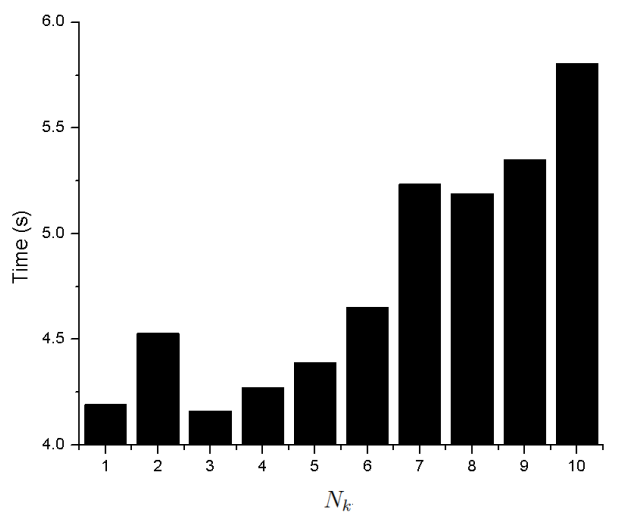

(b)

Figure 21: (a) Sensitivity analysis the MRK method with respect to $\lambda$ and $N_{k}$. (b) Average time required to perform the segmentation for different values of $N_{k}$. 


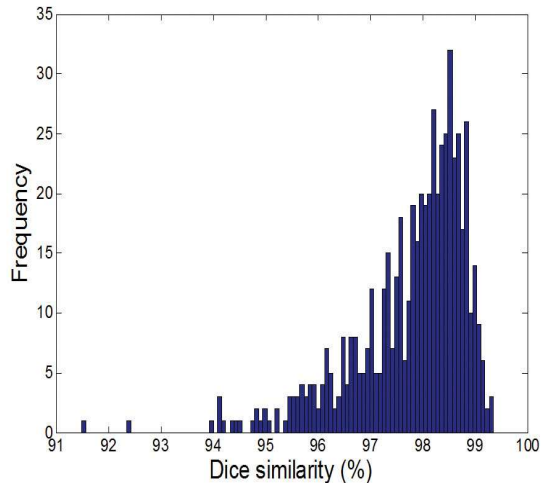

(a)

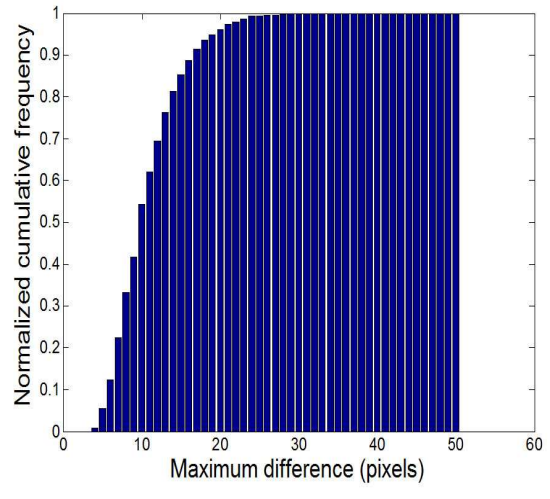

(b)

Figure 22: (a) Histogram of the Dice similarities, and (b) normalized cumulative histogram of the maximal radial lumen contour differences for consecutive frames. 


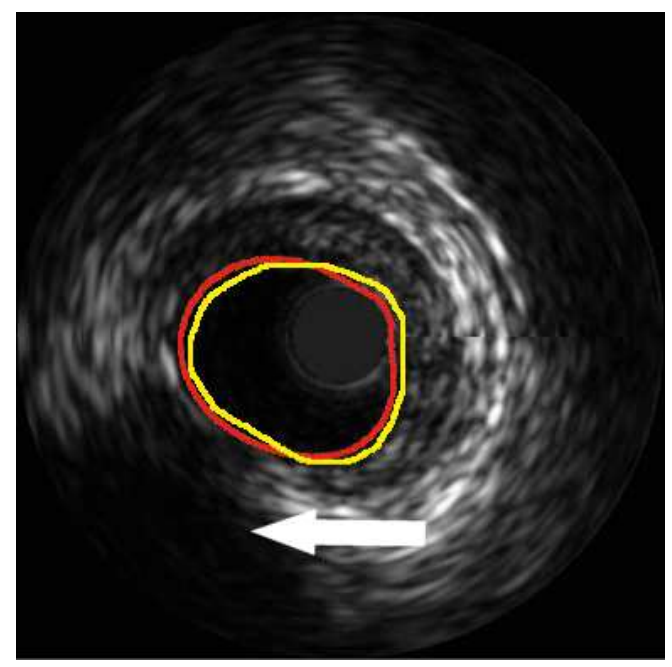

(a)

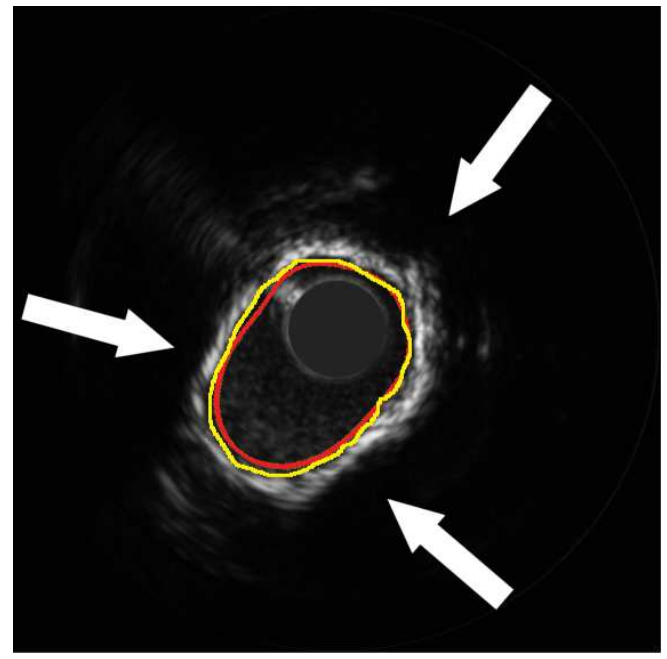

(c)

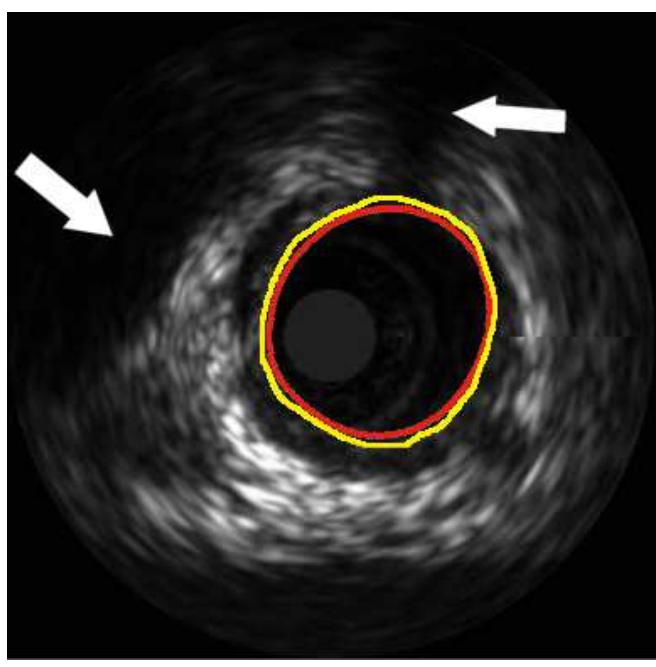

(b)

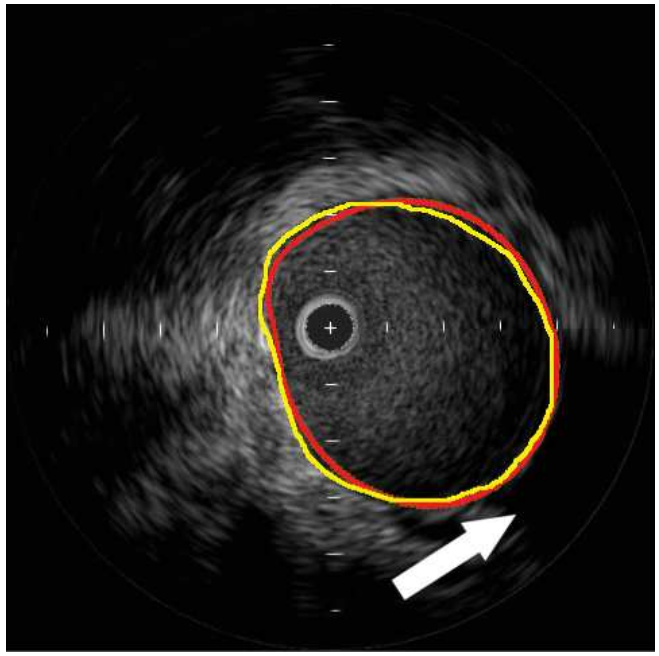

(d)

Figure 23: Segmentation results examples of (a, b, c) $20 \mathrm{MHz}$ and (c) $40 \mathrm{MHz}$ IVUS images with shadow artifacts. The arrows indicate the regions corresponding to shadows. The Dice similarity score for each frame is: (a) $93.78 \%$, (b) 94.72 , (c) $94.32 \%$, and (d) $95.64 \%$. 

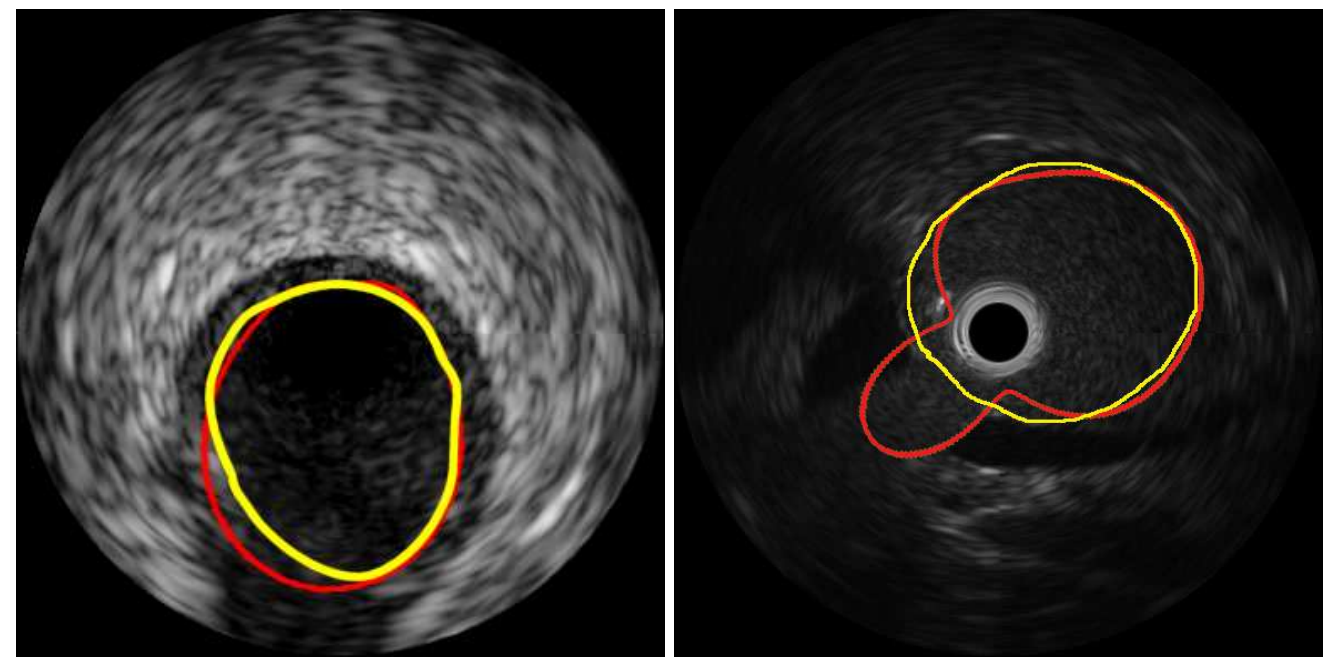

Figure 24: Segmentation results examples of (a) a $20 \mathrm{MHz}$ and (b) a $40 \mathrm{MHz}$ IVUS images depicting side branches. The Dice similarity score for each frame is: (a) $91.48 \%$ and (b) $86.66 \%$.

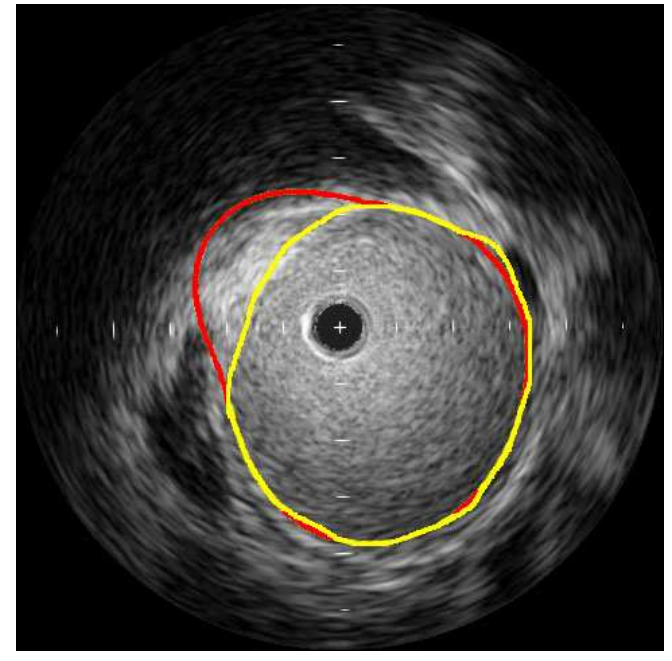

(a)

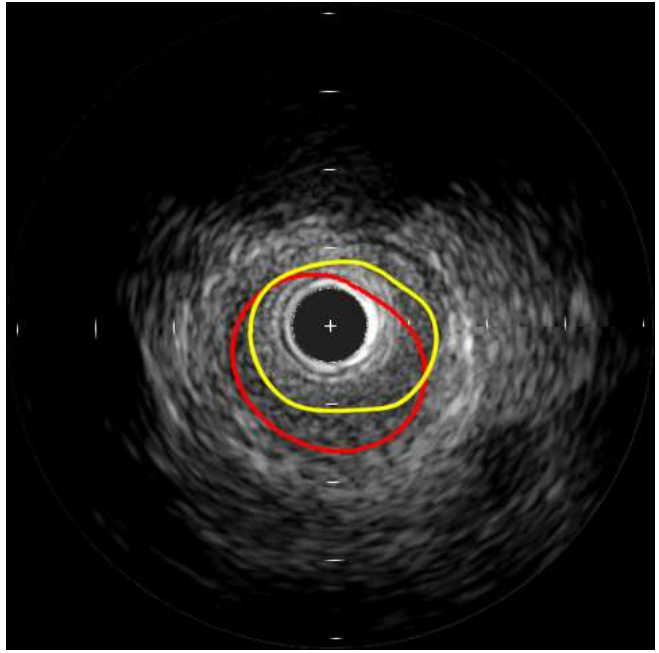

(b)

Figure 25: Example of two frames of a $40 \mathrm{MHz}$ sequence in which the texture of the lumen is similar to the texture of a region of the wall. The red line corresponds to the automatic segmentation, while the yellow line corresponds to the manual segmentation by the expert observer. The Dice similarity score for each frame is: (a) $88.72 \%$, (b) $77.23 \%$. 


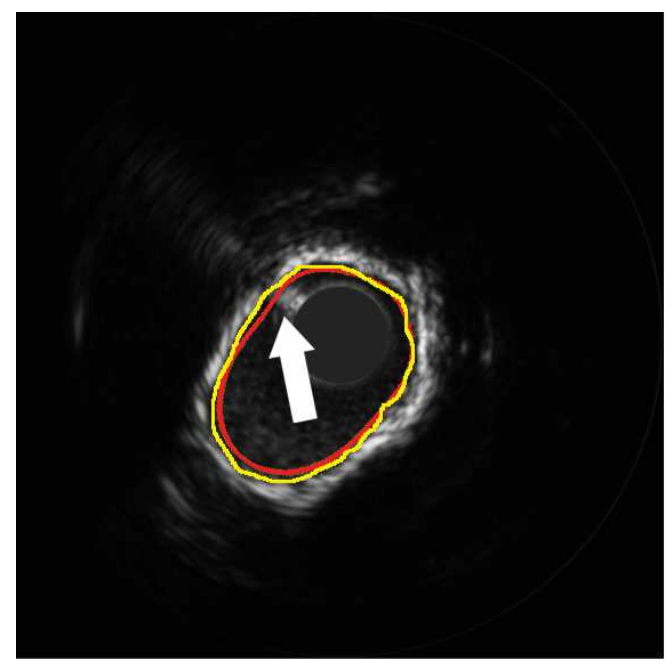

(a)

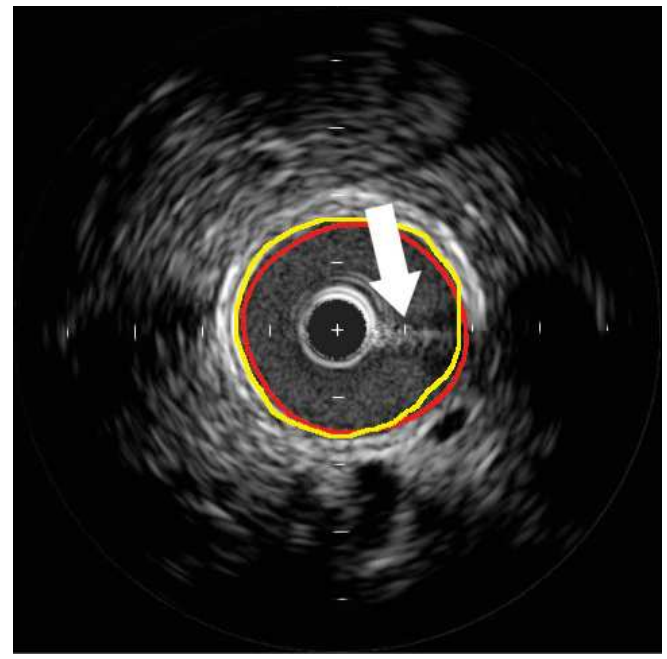

(c)

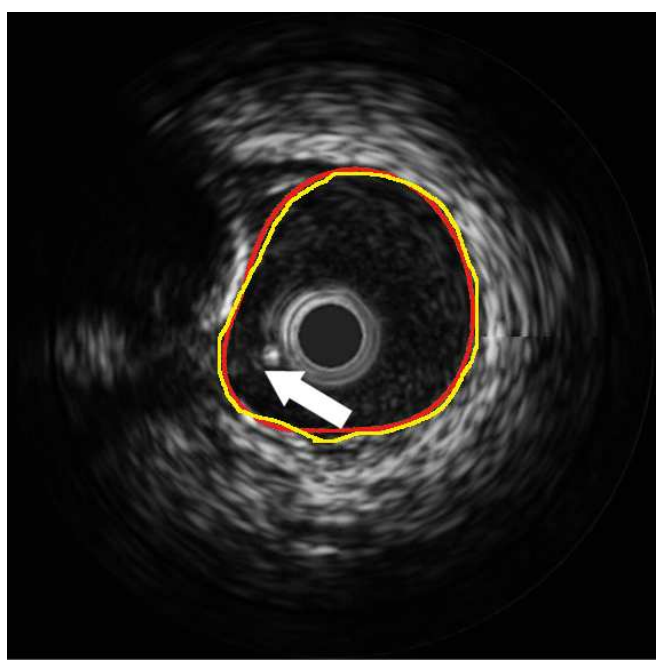

(b)

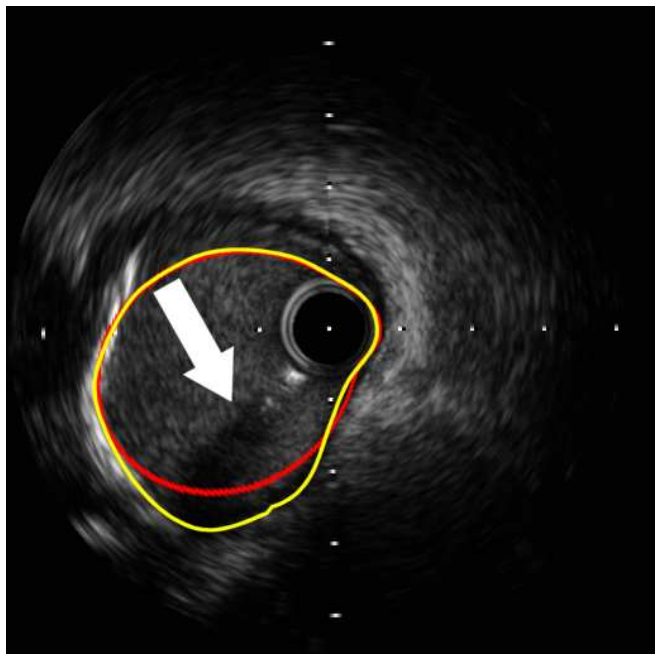

(d)

Figure 26: Segmentation results example of $40 \mathrm{MHz}$ IVUS images with guidewire artifacts. The arrows indicate the guidewire artifact. The Dice similarity score for each frame is: (a) $94.32 \%$, (b) $95.22 \%$, (c) $93.38 \%$ and (c) $93.38 \%$ and (d) $89.21 \%$. 


\subsection{Limitations}

Our method has three known limitations. The fist limitation is that it is not fully automatic since it requires samples from blood and non-blood regions in the first frame of the sequence. We could transform it to fully automatic by performing the computation of the SVM models using blood and non-blood samples from a large training data set of different IVUS images. However, the main limitation of fully automatic methods is that they rely on the use of large training sets for estimating the models of the gray intensity levels of the regions of interest. In order for these models to be robust, it would be necessary that this training database is large enough to account for all the different appearances that an IVUS image can have, including different visualization parameters.

The second limitation is its dependence on the accuracy of the annotations on the first frame of the sequence to segment. These samples should be representative of the different gray level intensities and texture of the regions of lumen and nonlumen. Incorrect samples may result in an incorrect segmentation. Therefore, this initialization must be performed by an observer familiarized with the IVUS data. It is recommended to select at least $50 \%$ of the region corresponding to lumen and most of the region adjacent to the lumen corresponding to non-lumen (Fig. 6).

The third limitation of our method relates with the inaccuracy of our method in the presence of artifacts in the IVUS images as discussed in Sec. 5.2

\section{Conclusion}

We have presented a probabilistic approach for the segmentation of the luminal border on IVUS images. The proposed method incorporates texture information by using the prediction of an SVM classifier model. This step enable the segmentation of IVUS images from different frequencies (i.e., 20 and $40 \mathrm{MHz}$ ) without the need of adjusting any parameter, and it makes it robust to the problem of variability of IVUS image B-mode reconstruction settings. The results indicate that the segmentation results obtained with our method are comparable with the segmentation performed by observers.

The robustness with respect to side branches by the prior detection of them (e.g., (Unal et al. (2008))), the exploration of additional texture descriptors and classification methods, the segmentation of the media/adventitia contour, and making the method fully automatic is subject to future work.

Acknowledgments: E.G. Mendizabal-Ruiz has been supported by a scholarship from CONACYT. M. Rivera has been supported in part by CONACYT grants 
131369, 169178, and 131771. I.A. Kakadiaris has been supported in part by NSF Grant DMS-0915242 and the UH Hugh Roy and Lillie Cranz Cullen Endowment Fund. Any opinions, findings, conclusions or recommendations expressed in this material are of the authors and may not reflect the views of the sponsors.

\section{References}

Balocco, S., Gatta, C., Ciompi, F., Pujol, O., Carrillo, X., Mauri, J., Radeva, P., June 2011. Combining growcut and temporal correlation for ivus lumen segmentation. In: Proc. $5^{\text {th }}$ Iberian Conference on Pattern Recognition and Image Analysis. Las Palmas de Gran Canaria, Spain, pp. 556 - 563.

Bland, J., Altman, D., Feb. 1986. Statistical methods for assessing agreement between two methods of clinical measurement. Lancet 1 (8476), 307-310.

Bovenkamp, E., Dijkstra, J., Bosch, J., Reiber, J., 2009. User-agent cooperation in multiagent IVUS image segmentation. IEEE Transactions on Medical Imaging 28 (1), 94-105.

Brusseau, E., de Korte, C. L., Mastik, F., Schaar, J., van der Steen, A. F. W., May 2004. Fully automatic luminal contour segmentation in intracoronary ultrasound imaging - a statistical approach. IEEE Transactions on Medical Imaging 23 (5), 554-566.

Burges, C. J. C., 1998. A tutorial on support vector machines for pattern recognition. Data Mining and Knowledge Discovery 2 (2), 121-167.

Cardinal, M., Meunier, J., Soulez, G., Maurice, R. L., Therasse, E., Cloutier, G., 2006. Intravascular ultrasound image segmentation: A three-dimensional fast-marching method based on gray level distributions. IEEE Transactions on Medical Imaging 25 (5), 590-601.

Cardinal, M., Soulez, G., Tardif, J. C., Meunier, J., Cloutier, G., 2010. Fastmarching segmentation of three-dimensional intravascular ultrasound images: A pre-and post-intervention study. Medical Physics 37 (7), 3633-3647.

Chang, C.-C., Lin, C.-J., 2011. LIBSVM: A library for support vector machines. ACM Transactions on Intelligent Systems and Technology 2 (3), 1-27. 
Ciompi, F., Pujol, O., Fernandez-Nofrerias, E., Mauri, J., Radeva, P., Sep. 20 - 24 2009. ECOC random fields for lumen segmentation in radial artery IVUS sequences. In: Proc. $12^{\text {th }}$ International Conference on Medical Image Computing and Computer Assisted Intervention. London, UK, pp. 869-876.

Dice, L., 1945. Measures of the amount of ecologic association between species. Ecology 26 (3), 297-302.

dos Santos Filho, E., Yoshizawa, M., Tanaka, A., Saijo, Y., 2006. A study on intravascular ultrasound image processing. Record of Electrical and Communication Engineering Conversazione, Tohoku University 74 (2), 30-33.

Downe, R., Wahle, A., Kovarnik, T., Skalicka, H., Lopez, J., Horak, J., Sonka, M., Sep. 10 2008. Segmentation of intravascular ultrasound images using graph search and a novel cost function. In: Proc. $2^{\text {nd }}$ MICCAI Workshop on Computer Vision for Intravascular and Intracardiac Imaging. New York, NY, pp. 71-79.

Duan, Q., Angelini, E. D., Laine, A. F., 2009. Surface function actives. Journal of Visual Communication and Image Representation 7 (20), 478 - 490.

Haas, C., Ermert, H., Holt, S., Grewe, P., Machraoui, A., Barmeyer, J., 2000. Segmentation of 3D intravascular ultrasonic images based on a random field model. Ultrasound in Medicine and Biology 26 (2), 297-306.

Hiro, T., Leung, C. Y., Russo, R. J., Karimi, H., Farvid, A. R., Tobis, J. M., 1996. Variability of a three-layered appearance in intravascular ultrasound coronary images: A comparison of morphometric measurements with four intravascular ultrasound systems. American Journal of Cardiac Imaging 10 (4), 219-227.

Hsu, C., Chang, C., Lin, C., July 2003. A practical guide to support vector classification. Tech. rep., National Taiwan University.

Hui-Zhu, H., Liang, Y., Friedman, M. H., January 2002. IVUS image segmentation based on contrast. In: Proc. SPIE Medical Imaging: Image Processing. Vol. 4684. San Jose, California, USA, pp. 1727-1733.

Katouzian, A., Angelini, E. D., Sturm, B., Laine, A. F., May 2012. Brushlet-driven segmentation framework for automatic detection of lumen borders in ivus images with comparison study. In: Proc. International Symposium on Biomedical Imaging. Barcelona, Spain., pp. $242-245$. 
Katouzian, A., Baseri, B., Konofagou, E., Laine, A., February 2008. Automatic detection of blood versus non-blood regions on intravascular ultrasound (IVUS) images using wavelet packet signatures. In: Proc. SPIE Medical Imaging: Ultrasonic Imaging and Signal Processing. San Diego, CA, pp. $1-8$.

Kerridge, D. F., 1961. Inaccuracy and inference. Journal of the Royal Statistical Society 23 (1), $184-194$.

Klingensmith, J. D., Shekhar, R., Vince, D. G., 2000. Evaluation of threedimensional segmentation algorithms for the identification of luminal and medial-adventitial borders in intravascular ultrasound images. IEEE Transactions on Medical Imaging 19 (10), 996-1011.

Kovalski, G., Beyar, R., Shofti, R., Azhari, H., 2000. Three-dimensional automatic quantitative analysis of intravascular ultrasound images. Ultrasound in Medicine and Biology 26 (4), 527-537.

Laws, K. I., July 1980. Rapid texture identification. In: Proc. SPIE Conference on Image Processing for Missile Guidance. San Diego, CA, pp. 376-380.

Lee, D. C., Schenk, T., August 1992. Image segmentation from texture measurement. ISPRS Journal of Photogrammetry and Remote Sensing XXIX, Part B3, Commission III, 195-199.

Lloyd-Jones, D., Adams, R., Carnethon, M., De Simone, G., Ferguson, T. B., Flegal, K., Ford, E., Furie, K., Go, A., Greenlund, K., Haase, N., Hailpern, S., Ho, M., Howard, V., Kissela, B., Kittner, S., Lackland, D., Lisabeth, L., Marelli, A., McDermott, M., Meigs, J., Mozaffarian, D., Nichol, G., O’Donnell, C., Roger, V., Rosamond, W., Sacco, R., Sorlie, P., Stafford, R., Steinberger, J., Thom, T., Wasserthiel-Smoller, S., Wong, N., Wylie-Rosett, J., Hong, Y., 2009. Heart disease and stroke statistics-2009 update: A report from the American Heart Association statistics committee and stroke statistics subcommittee. Circulation 119 (3), 480-486.

Luo, Z., Wang, Y., Wang, W., Apr. 2003. Estimating coronary artery lumen area with optimization-based contour detection. IEEE Transactions on Medical Imaging 22 (4), 564 - 566.

Meier, D. S., Cothren, R. M., Vince, D. G., Cornhill, J. F., Jun. 1997. Automated morphometry of coronary arteries with digital image analysis of intravascular ultrasound. American Heart Journal 133 (6), 681-690. 
Mendizabal-Ruiz, E. G., Rivera, M., Kakadiaris, I. A., Jun. 24-26 2008. A probabilistic segmentation method for the identification of luminal borders in intravascular ultrasound images. In: Proc. IEEE Computer Society Conference on Computer Vision and Pattern Recognition. Anchorage, AK, pp. 1-8.

Mintz, G., Nissen, S., Anderson, W., Bailey, S., Erbel, R., Fitzgerald, P., Pinto, F., Rosenfield, K., Siegel, R., Tuzcu, E., Yock, P., 2001. American College of Cardiology clinical expert consensus document on standards for acquisition, measurement and reporting of intravascular ultrasound studies (IVUS). Journal of the American College of Cardiology 37 (5), 1478-1492.

Mojsilovic, A., Popovic, M., Amodaj, N., Babic, R., Ostojic, M., Nov. 1997. Automatic segmentation of intravascular ultrasound images: A texture-based approach. Annals of Biomedical Engineering 25 (6), 1059-1071.

Moraes, M., Furuie, S., 2011. Automatic coronary wall segmentation in intravascular ultrasound images using binary morphological reconstruction. Ultrasound in Medicine and Biology 37 (9), 1486-1499.

Nissen, S. E., Yock, P., January 2001. Intravascular ultrasound: Novel pathophysiological insights and current clinical applications. Circulation 103 (4), 604616.

Nocedal, J., Wright, S. J., 1999. Numerical Optimization. Springer.

Papadogiorgaki, M., Mezaris, V., Chatzizisis, Y. S., Giannoglou, G. D., Kompatsiaris, I., Sep. 2008. Image analysis techniques for automated IVUS contour detection. Ultrasound in Medicine and Biology 34 (9), 1482-1498.

Plissiti, M. E., Fotiadis, D. I., Michalis, L. K., Bozios, G. E., Jun. 2004. An automated method for lumen and media-adventitia border detection in a sequence of IVUS frames. IEEE Transactions on Information Technology in Biomedicine 8 (2), 131-141.

Rivera, M., Dalmau, O., 2012. Variational viewpoint of the quadratic markov measure field models: theory and algorithms. IEEE Transactions on Image Processing 21 (3), $1246-1257$.

Sonka, M., Zhang, X., Siebes, M., Bissing, M. S., DeJong, S. C., Collins, S. M., McKay, R. C., Dec. 1995. Segmentation of intravascular ultrasound images: 
A knowledge-based approach. IEEE Transactions on Medical Imaging 14 (4), 719-732.

Taki, A., Najafi, Z., Roodaki, A., Setarehdan, S. K., Zoroofi, R. A., Konig, A., Navab, N., 2008. Automatic segmentation of calcified plaques and vessel borders in IVUS images. International Journal of Computer Assisted Radiology and Surgery 3 (3-4), $347-354$.

Unal, G., Bucher, S., Carlier, S., Slabaugh, G., Fang, T., Tanaka, K., May 2008. Shape-driven segmentation of the arterial wall in intravascular ultrasound images. IEEE Transactions on Information Technology in Biomedicine 12 (3), 335-347.

von Birgelen, C., Mario, C. D., Li, W., Schuurbiers, J. C. H., Slager, C. J., de Feyter, P. J., Roelandt, J. R. T. C., Serruys, P. W., 1996. Morphometric analysis in three-dimensional intracoronary ultrasound: An in vitro and in vivo study using a novel system for the contour detection of lumen and plaque. American Heart Journal 132 (3), 516-527.

Wennogle, M., Hoff, W., July 2009. Three dimensional segmentation of intravascular ultrasound data. In: Proc. $6^{\text {th }}$ International Conference on Image Analysis and Recognitio. Vol. 5627. Toronto, Canada, pp. 772-781.

Wu, T., Lin, C., Weng, R., 2004. Probability estimates for multi-class classification by pairwise coupling. Journal of Machine Learning Research 5, 975-1005.

Zhang, X., McKay, C. R., Sonka, M., Dec. 1998. Tissue characterization in intravascular ultrasound images. IEEE Transactions on Medical Imaging 17 (6), 889-899.

Zhu, X., Zhangc, P., Shaoa, J., Chenga, Y., Zhangc, Y., Bai, J., 2011. A snakebased method for segmentation of intravascular ultrasound images and its in vivo validation. Ultrasonics 51 (2), 181-189. 